\title{
CONTRIBUTION TO THE KNOWLEDGE OF THE LICHEN BIOTA OF BOLIVIA. 7
}

\author{
Adam Flakus, Harrie J. M. Sipman, Pamela Rodriguez Flakus, \\ AgnieszKa Jablońska, Magdalena Oset, Rosa I. Meneses Q. \\ \& MARTIN KUKWA ${ }^{1}$
}

\begin{abstract}
This paper presents new distribution records for 80 lichen taxa from Bolivia, including 14 new national records: Bulbothrix thomasiana Benatti \& Marcelli, Dirinaria confluens (Fr.) Awasthi var. coccinea (Lynge) Awasthi, D. melanocarpa (Müll. Arg.) C. W. Dodge, Gyalideopsis lambinonii Vězda, Malmidea sulphureosorediata M. Cáceres, D. A. Mota \& Aptroot, Parmelia warmingii Vain., Ramalina aspera Räsänen, R. canaguensis V. Marcano \& A. Morales, R. disparata Krog \& Swinscow, $R$. grumosa Kashiw., $R$. incana Kashiw., R. puiggarii Müll. Arg., R. usnea (L.) Howe and Ramonia valenzueliana (Mont.) Stizenb. Notes on distribution and chemistry are provided for most species.
\end{abstract}

Key words: biodiversity, biogeography, lichenized fungi, Neotropics, South America

Adam Flakus, Laboratory of Lichenology, W. Szafer Institute of Botany, Polish Academy of Sciences, Lubicz 46, 31-512 Kraków, Poland; e-mail: a.flakus@botany.pl

Harrie J. M. Sipman, Botanischer Garten \& Botanisches Museum Berlin Dahlem, Königin-Luise-Strasse 6-8, D-14195 Berlin, Germany

Pamela Rodriguez Flakus, Department of Botany and Molecular Evolution, Senckenberg Forschungsinstitut und Naturmuseum, Senckenberganlage 25, D-60325, Frankfurt am Main, Germany; Herbario Nacional de Bolivia, Instituto de Ecología, Universidad Mayor de San Andrés, Calle 27, Cota Cota, Casilla 10077, La Paz, Bolivia

Agnieszka Jabłońska, Martin Kukwa \& Magdalena Oset, Department of Plant Taxonomy and Nature Conservation, University of Gdańsk, Wita Stwosza 59,80-308 Gdańsk, Poland; e-mail: dokmak@ug.edu.pl

Rosa I. Meneses Q., Herbario Nacional de Bolivia, Agreement Instituto de Ecología - Museo Nacional de Historia Natural, Calle 27, Cota Cota, Casilla 10077, La Paz, Bolivia

\section{INTRODUCTION}

Bolivia preserves the most diverse mosaic of vegetation in South America (Josse et al. 2003), including part of the tropical Andes hotspot (Myers et al. 2000). Thus the country offers an extraordinarily wide variety of potential habitats for lichens, still in need of further investigation. This, our seventh contribution to knowledge of the diversity and distribution of lichenized fungi of Bolivia, presents data based on recent collections made by the authors, complemented by further revision of various herbarium specimens. New distribution records are given, and several species are reported for the first time from the country.

\footnotetext{
1 Corresponding author
}

\section{MATERIAL AND METHODS}

The examined specimens are housed at B, BM, BOLV, KRAM, LPB, UGDA (acronyms after Thiers 2012) and the private herbarium of A. Flakus, with duplicates deposited in other herbaria. Secondary lichen metabolites were identified by thin-layer chromatography (TLC) following Culberson and Kristinsson (1970) and Orange et al. (2001). Thallus fluorescence under ultraviolet light (UV) and reactions with $\mathrm{K}(10 \%$ water solution of potassium hydroxide) and $\mathrm{C}$ (commercial bleach) were checked in some species. Notes on general distribution are presented for species noted as new for Bolivia.

The following abbreviations are used: $A F-\mathrm{A}$. Flakus, $J Q-\mathrm{J}$. Quisbert, $K B-\mathrm{K}$. Bach, $M K-\mathrm{M}$. Kukwa, $O P-$ O. Plata, $P R-$ P. Rodriguez Flakus, $R I S-\mathrm{R}$. I. Meneses; AMBORÓ - Parque Nacional y Área Natural de Manejo 
Integrado Amboró; APOLOBAMBA - Área Natural de Manejo Integrado Nacional Apolobamba; CARRASCO Parque Nacional Carrasco; COTAPATA - Parque Nacional y Área Natural de Manejo Integrado Cotapata; KAA-IYA - Parque Nacional y Área Natural de Manejo Integrado Kaa-Iya del Gran Chaco; SAJAMA - Parque Nacional Sajama; TARIQUÍA - Reserva Nacional de Flora y Fauna Tariquía (abbreviations of protected areas according to SERNAP Bolivia; http://www.sernap.gob.bo).

Species noted as new for Bolivia are asterisked ( ${ }^{*}$ ), and $( \pm)$ indicates that some specimens lacked a particular substance.

\section{RESULTS}

Alectoria ochroleuca (Hoffm.) A. Massal.

This species has only rarely been reported from Bolivia (Nylander 1861; Herzog 1922; Feuerer et al. 1998; Flakus et al. 2012a).

Specimen examined: BOliVia. Dept. La Paz. Prov. Murillo, oberhalb Aldea Taquesi, 4500 m, on rock, 6 Apr. 1987, S. Stab LB-57 (B).

\section{*Bulbothrix thomasiana Benatti \& Marcelli}

So far known from Brazil, French Guyana and Venezuela (Marcelli et al. 2011).

Chemistry. Atranorin and lobaric acid.

Specimens examined: BOliVia. Dept. La Paz. Prov. Abel Iturralde, forest above Tumupasa village, $14^{\circ} 08^{\prime} 51^{\prime \prime} \mathrm{S}, 67^{\circ} 53^{\prime} 34^{\prime \prime} \mathrm{W}$, alt. $350 \mathrm{~m}$, Subandean Amazon forest, on bark of tree, 31 Aug. 2008, $M K 7015$ (LPB, UGDA).

\section{Candelaria fibrosa (Fr.) Müll. Arg.}

The species was reported from Bolivia only by Poelt (1974) and Feuerer et al. (1998)

Specimens examined: BOliVia. Dept. La Paz. Prov. Murillo, La Paz, Laguna de Cota-Cota (calle 31), $3750 \mathrm{~m}, 16^{\circ} 32.4^{\prime} \mathrm{S}, 68^{\circ} 04.0^{\prime} \mathrm{W}$, Puna y vegetacion altoandina, on Ulmus sp., 8 Feb. 2002, A. D. CansecoTarifa 107 (B, LPB).

\section{Cladia aggregata (Sw.) Nyl.}

The species is rather common in Bolivia and especially abundant in cloud forests and Páramo Yungeño (Flakus et al. 2012a).
Specimens examined: BOliVia. Dept. La Paz. Prov. Murillo, Tres Rios, Cordillera Real, 4100 m, 4 July 1973, G. Frey 1-1309 (B); Prov. Nor Yungas, Coscapa, ca $4 \mathrm{~km}$ de Cotapata hacia La Paz. $3450 \mathrm{~m}$, $16^{\circ} 23^{\prime} \mathrm{S}, 67^{\circ} 53^{\prime} \mathrm{W}$, pastizal con rocas, terricolous, 10 Sept. 1997, KB et al. 485 (B, LPB); $5 \mathrm{~km} \mathrm{del}$ camino principal de Chuspipata hacia Coroico, $2700 \mathrm{~m}$, $16^{\circ} 23^{\prime} \mathrm{S}, 67^{\circ} 48^{\prime} \mathrm{W}$, terricolous, 19 Sept. 1997, KB et al. 549 (B, LPB).

\section{Cladonia didyma (Fée) Vain.}

This species is one of the commonest members of the genus in Bolivia (Flakus et al. 2012a and literature cited therein).

Specimens examined: BOliVia. Dept. CochaBAmba. Prov. Ayopaya, $10 \mathrm{~km}$ de Cocapata hacia Cotacajes, $2750-2850 \mathrm{~m}, 16^{\circ} 38^{\prime} \mathrm{S}, 66^{\circ} 41^{\prime} \mathrm{W}$, bosque húmedo, terricolous, 10 \& 12 May 1997, KB et al. $5 \& 49$ (B, LPB); Dept. La Paz. Prov. Bautista Saavedra, $3 \mathrm{~km}$ de Pauji-Yuyo hacia Charazani, $1300 \mathrm{~m}, 15^{\circ} 02^{\prime} \mathrm{S}, 68^{\circ} 29^{\prime} \mathrm{W}$, bosque siempreverde virgen, epiphyte, 5 June 1997, KB et al. 138 (B, LPB); 6 km de Pauji-Yuyo hacia Charazani, $1350 \mathrm{~m}$, $15^{\circ} 02^{\prime} \mathrm{S}, 68^{\circ} 29^{\prime} \mathrm{W}$, bosque siempreverde, decaying wood, 14 June 1997, KB et al. 162 (B, LPB); Cerro Asunta Pata, $1500 \mathrm{~m}, 15^{\circ} 05^{\prime} \mathrm{S}, 68^{\circ} 29^{\prime} \mathrm{W}$, pared lodosa, terricolous, 21 June 1997, KB et al. 171, 172, 173 \& 176 (B, LPB); ibid., bosque siempreverde, decaying wood, 21 June 1997, KB et al. 185 (B, LPB); $15 \mathrm{~km}$ de Charazani hacia Apolo, $2450 \mathrm{~m}, 15^{\circ} 11^{\prime} \mathrm{S}, 68^{\circ} 52^{\prime} \mathrm{W}$, bosque húmedo secundario, base of tree, 2 July 1997, $K B$ et al. 310 (B, LPB); Prov. Nor Yungas, Cotapata, between Sandillani and Bajo Hornuni, $1520 \mathrm{~m}$, $16^{\circ} 11.1^{\prime} \mathrm{S}, 67^{\circ} 49.5^{\prime} \mathrm{W}$, on bark, 13 Oct. $2006, P R$ et al. 142 (B, LPB); Serranía Bellavista, ca $43 \mathrm{~km}$ por camino de Caranavi hacia Sapecho, $1400 \mathrm{~m}, 15^{\circ} 41^{\prime} \mathrm{S}$, $67^{\circ} 30^{\prime} \mathrm{W}$, borde del camino, decaying wood, $27 \mathrm{Aug}$. 1997, KB et al. 424 (B, LPB, as admixture to C. subradiata).

\section{Dibaeis columbiana (Vain.) Kalb \& Gierl}

This species is known in Bolivia from only a few records (Flakus et al. 2012a and literature cited therein).

Specimens examined: BOliVia. Dept. La Paz. Prov. Nor Yungas, Coscapa, senda al oueste del camino principal ca $4 \mathrm{~km}$ de Cotapata hacia La Paz, $3300 \mathrm{~m}$, $16^{\circ} 23^{\prime} \mathrm{S}, 67^{\circ} 53^{\prime} \mathrm{W}$, pared rocosa, on rock, 13 Sept. 1997 , $K B$ et al. 521 (B, LPB). 
${ }^{*}$ Dirinaria confluens (Fr.) Awasthi var. coccinea (Lynge) Awasthi

This variety has been reported so far from Brazil, Paraguay and Venezuela (Awasthi 1975; López-Figueiras 1986).

Chemistry. Atranorin and divaricatic acid; red pigment in medulla.

Specimen examined: BoliVia. Dept. La Paz. Prov. Nor Yungas, near Pongo village, $16^{\circ} 19^{\prime} 28^{\prime \prime}$ S, $67^{\circ} 57^{\prime} 21^{\prime \prime} \mathrm{W}, 3822 \mathrm{~m}$, Páramo Yungueño, on twig, 26 Nov. 2011, MK 10456c (LPB).

*Dirinaria melanocarpa (Müll. Arg.) C. W. Dodge

This is a Neotropical taxon known from Brazil, Colombia and Paraguay (Awasthi 1975; Sipman et al. 2008).

Chemistry. Atranorin and divaricatic acid.

Specimen examined: BoliVia. Dept. La Paz. Prov. Nor Yungas, near Pongo village, $16^{\circ} 19^{\prime} 28^{\prime \prime}$, $67^{\circ} 57^{\prime} 21^{\prime \prime} \mathrm{W}, 3822 \mathrm{~m}$, Páramo Yungueño, on twig, 26 Nov. 2011, MK 10456b (LPB).

\section{Flavoparmelia gerlachei (Zahlbr.) Hale}

Reported in Bolivia only by Feuerer et al. (1998) and Quilhot et al. (2007).

Chemistry. Usnic and protocetraric acids.

Specimens examined: BoliVia. Dept. Potosí. Prov. Nor Lipez, Viscachitas near el Arbol del Piedra, $21^{\circ} 55^{\prime} 33^{\prime \prime} \mathrm{S}, 68^{\circ} 00^{\prime} 18^{\prime \prime} \mathrm{W}, 4612 \mathrm{~m}$, high Andean open semi-desert, saxicolous, 5 Dec. 2009, AF \& PR 14736.19 (KRAM, LPB).

\section{Flavopunctelia soredica (Nyl.) Hale}

This is the second locality of the species in Bolivia (Flakus et al. 2012a).

Chemistry. Usnic and lecanoric acids.

Specimens examined: BOlivia. Dept. La Paz. Prov. Omasuyos, El Dragon hill near Chahualla, $15^{\circ} 51^{\prime} 17^{\prime \prime} \mathrm{S}, 69^{\circ} 00^{\prime} 40^{\prime \prime} \mathrm{W}, 3850 \mathrm{~m}$, Puna Húmeda vegetation, on rock, 19 May 2011, MK 8982 (LPB, UGDA).

\section{${ }^{*}$ Gyalideopsis lambinonii Vězda}

The species has been reported in the Neotropics from Brazil, Colombia, Costa Rica and the USA (Florida), and elsewhere from Africa (Rwanda \& Zaire), Australia and Taiwan (Vězda 1979; Kalb \& Vězda 1988; Kalb 1996; Aptroot \& Sparrius 2003; Breuss \& Neuwirth 2007; Lücking et al. 2007; Sipman et al. 2008).

Specimen examined: BOliVia. Dept. La Paz. Prov. Franz Tamayo, near Yuyo village, $15^{\circ} 03^{\prime} 30^{\prime \prime}$, $68^{\circ} 19^{\prime} 24^{\prime \prime} \mathrm{W}, 1230 \mathrm{~m}$, Preandean Amazon forest, on bark, 23 Nov. 2011, MK 10365 (LPB).

\section{Heterodermia arvidssonii Moberg}

This is the third locality of the species in Bolivia (Flakus et al. 2013).

Specimens examined: BOliVia. Dept. La Paz. Prov. Nor Yungas, Coroico, near Sol y Luna hotel, $1870 \mathrm{~m}, 16^{\circ} 11^{\prime} 56^{\prime \prime} \mathrm{S}, 67^{\circ} 43^{\prime} 26^{\prime \prime} \mathrm{W}$, gardens with trees, on Citrus sp., 13 Dec. 2011, MK 10764 (LPB, UGDA; as admixture to Herpothallon rubrocinctum).

Heterodermia casarettiana (A. Massal.) Trevis.

It was only rarely reported from Bolivia (Flakus et al. 2012a and literature cited therein) but appears to be common there.

Specimens eXamined: BOLIVIA. Dept. CochaBamba. Prov. Ayopaya, $20 \mathrm{~km}$ de Cocapata hacia Cotacajes, $2000 \mathrm{~m}, 16^{\circ} 46^{\prime} \mathrm{S}, 66^{\circ} 44^{\prime} \mathrm{W}$, W-exposicion, bosque semideciduo disturbado de $5 \mathrm{~m}$ de altura, on rock, 15 May 1997, KB et al. 101 (B, LPB); comunidad de Saila Pata, $3150 \mathrm{~m}, 16^{\circ} 54^{\prime} \mathrm{S}, 66^{\circ} 56^{\prime} \mathrm{W}$, S-exposicion, bosque siempreverde, epiphyte, 14 Nov. 1997, KB \& I. Jimenez 906 (B, LPB); Prov. Chapare, CARRASCO, $130 \mathrm{~km}$ del camino antiguo de Cochabamba a Villa Tunari, $2200 \mathrm{~m}$, $17^{\circ} 07^{\prime} \mathrm{S}, 65^{\circ} 36^{\prime} \mathrm{W}$, bosque húmedo con paredes rocosas, terricolous, 10 Oct. 1997, KB et al. 692 (LPB); $156 \mathrm{~km}$ del camino antiguo de Cochabamba a Villa Tunari, $850 \mathrm{~m}, 17^{\circ} 05^{\prime} \mathrm{S}, 65^{\circ} 32^{\prime} \mathrm{W}$, bosque siempreverde, epiphyte, 12 Oct. 1997, KB et al. 843a (B, LPB); DеPт. LA Paz. Prov. Bautista Saavedra, $15 \mathrm{~km}$ de Camata hacia Apolo, $1400 \mathrm{~m}, 15^{\circ} 13^{\prime} \mathrm{S}, 68^{\circ} 41^{\prime} \mathrm{W}$, bosque semideciduo, on rock, 27 June 1997, KB et al. 227 (B, LPB); Prov. Nor Yungas, cerca Cotapata, $1490 \mathrm{~m}, 16^{\circ} 11.1^{\prime} \mathrm{S}$, $67^{\circ} 49.5^{\prime} \mathrm{W}$, bosque muy denso, 14 May 2006, PR et al. 113 (B, LPB); Prov. Sud Yungas, Alto Beni Sapecho, Colonia Tupiza, $750 \mathrm{~m}, 15^{\circ} 31^{\prime} \mathrm{S}, 67^{\circ} 17^{\prime} \mathrm{W}$, bosque 
siempreverde a semideciduo, epiphyte, 2 July 1999, A. Acebey \& T. Krömer 935 (B, LPB); Cieneguillas, $1300 \mathrm{~m}, 16^{\circ} 35^{\prime} \mathrm{S}, 67^{\circ} 26^{\prime} \mathrm{W}$, bosque deciduo, epiphyte, 14 Dec. 1997, KB et al. 926 (B, LPB); DePt. SANTA Cruz. Prov. Florida, Refugio Los Volcanes, $1000 \mathrm{~m}$, $18^{\circ} 06^{\prime} \mathrm{S}, 63^{\circ} 36^{\prime} \mathrm{W}$, on rock, 6 Oct. $1997, \mathrm{~KB}$ et al. 650 (B, LPB, as admixture to Heterodermia obscurata).

\section{Heterodermia corallophora (Tayl.) Skorepa}

Only recently reported for the first time from Bolivia (Flakus et al. 2012a).

Specimens examined: BOLIVIA. Dept. CochaBAmba. Prov. Ayopaya, $10 \mathrm{~km}$ de Cocapata hacia Cotacajes, $2750 \mathrm{~m}, 16^{\circ} 38^{\prime} \mathrm{S}, 66^{\circ} 41^{\prime} \mathrm{W}$, bosque húmedo, decaying wood, 12 May 1997, KB et al. 51 (B, LPB); $5 \mathrm{~km}$ al sur de Saila Pata. $3050 \mathrm{~m}, 16^{\circ} 55^{\prime} \mathrm{S}, 66^{\circ} 56^{\prime} \mathrm{W}$, bosque siempreverde, epiphyte, 10 Nov. 1997, KB \& I. Jimenez 859 (B, LPB); Dept. La Paz. Prov. Bautista Saavedra, $15 \mathrm{~km}$ de Charazani hacia Apolo, $2400 \mathrm{~m}, 15^{\circ} 11^{\prime} \mathrm{S}, 68^{\circ} 52^{\prime} \mathrm{W}$, pastos con arbustos al lado del Río Camata, terricolous, 1 July 1997, KB et al. 261 (B, LPB).

\section{Heterodermia lutescens (Kurok.) Follman}

Previously reported from Bolivia only by Moberg (2011) and Flakus et al. (2012a).

Specimens examined: BOliVia. Dept. La Paz. Prov. Nor Yungas, Coroico, near Sol y Luna hotel, $1870 \mathrm{~m}, 16^{\circ} 11^{\prime} 56^{\prime \prime} \mathrm{S}, 67^{\circ} 43^{\prime} 26^{\prime \prime} \mathrm{W}$, gardens with trees, on Citrus sp., 13 Dec. 2011, MK 10764 (LPB, UGDA; as admixture to Herpothallon rubrocinctum).

\section{Heterodermia obscurata (Nyl.) Trevis.}

This species was reported from Bolivia only by Feuerer and Sipman (2005).

Specimens examined: BOLIVIA. Dept. CochaBAmBa. Prov. Ayopaya, $10 \mathrm{~km}$ de Cocapata hacia Cotacajes, $2750 \mathrm{~m}, 16^{\circ} 38^{\prime} \mathrm{S}, 66^{\circ} 41^{\prime} \mathrm{W}$, bosque húmedo, epiphyte, 12 May 1997, KB et al. 52 (LPB); DePT. LA PAz. Prov. Bautista Saavedra, $15 \mathrm{~km}$ de Camata hacia Apolo, $1300 \mathrm{~m}, 15^{\circ} 13^{\prime} \mathrm{S}, 68^{\circ} 41^{\prime} \mathrm{W}$, bosque semi-deciduo, epiphyte, 24 June 1997, KB et al. 211 (LPB); DePt. SANTA CruZ. Prov. Florida, Refugio Los Volcanes, $1000 \mathrm{~m}, 18^{\circ} 06^{\prime} \mathrm{S}, 63^{\circ} 36^{\prime} \mathrm{W}$, bosque semi-deciduo, terricolous, 3 Oct. 1997, KB et al. 629 (B, LPB); ibid., ladera arenosa de una pared rocosa, on rock, 6 Oct. 1997, KB et al. 650 (B, LPB).
Hypotrachyna cirrhata (Fr.) Divakar, A. Crespo, Sipman, Elix \& Lumbsch

Everniastrum cirrhatum (Fr.) Sipman

The species appears to be common in Bolivia but in some provinces it has not been noted or was reported from single localities (Flakus et al. 2011b).

Specimens examined: BOliVia. Dept. La Paz. Prov. Nor Yungas, near Chuspipata, $16^{\circ} 17^{\prime} 09^{\prime \prime} \mathrm{S}$, $67^{\circ} 51^{\prime} 00^{\prime \prime} \mathrm{W}, 3228 \mathrm{~m}$, Yungas cloud forest, on saxicolous bryophytes, 24 May 2011, MK 9282 (LPB, UGDA); Prov. Sud Yungas, Mururata, bajo la Mina Bolsa Negra, $3910 \mathrm{~m}, 16^{\circ} 35^{\prime} \mathrm{S}, 67^{\circ} 45^{\prime} \mathrm{W}$, pastizal semialto, on rock, 27 Feb. 1995, J. Gonzáles 221 (B, LPB); DePt. SANTA Cruz, Prov. Florida, AMBORÓ, above la Yunga village,

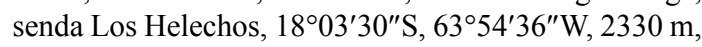
Yungas cloud forest, on terricolous bryophytes, 7 June 2011, MK 9773 (LPB, UGDA).

Hypotrachyna sorocheila (Vain.) Divakar, A. Crespo, Sipman, Elix \& Lumbsch

Everniastrum sorocheilum (Vain.) Sipman

This is only the third locality of the species from Bolivia (Flakus et al. 2011b).

Specimens examined: BOLIVIA. Dept. Tarija. Prov.

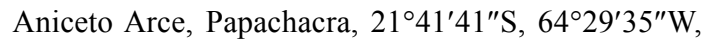
$2170 \mathrm{~m}$, Tucumano-Boliviano montano forest, on bark, 7 Aug. 2012, MK 11034 (LPB, UGDA).

Hypotrachyna subformosana Hale ex Elix, T. H. Nash \& Sipman

This is the second record of the species in Bolivia (Flakus et al. 2012b).

Chemistry. Lichexanthone, lividic acid and related substances in minor to trace amounts.

Specimen examined: BOliVia. Dept. La Paz. Prov. Franz Tamayo, between Apolo and Mapiri, $14^{\circ} 50^{\prime} 51^{\prime \prime} \mathrm{S}, 68^{\circ} 21^{\prime} 38^{\prime \prime} \mathrm{W}, 1490 \mathrm{~m}$, savannah with scattered trees, shrubs, along streams, on twig, 23 Nov. 2011, MK 10268a (LPB).

\section{Josefpoeltia parva (Räsänen) Frödén} \& L. Lindblom

Josefpoeltia boliviensis S. Y. Kondr. \& Kärnefelt 
Rarely reported from Bolivia (Kondratyuk \& Kärnefelt 1997; Frödén \& Lindblom 2003; Canseco et al. 2006).

Specimen examined: BOlivia. Dept. La Paz, Jupopina, 3400 m, on Prosopis, 12 Feb. 1987, S. Stab $L B-10$ (B).

\section{Lepraria achariana Flakus \& Kukwa}

In Bolivia previously reported from only five localities (Flakus \& Kukwa 2007; Flakus et al. 2011a).

Chemistry. Lecanoric acid as main lichen metabolite.

Specimens examined: BOLIVIA. Dept. LA Paz. Prov. Nor Yungas, COTAPATA, by Sillu Tincara pre-Columbian route, $16^{\circ} 17^{\prime} 38^{\prime \prime} \mathrm{S}, 67^{\circ} 53^{\prime} 33^{\prime \prime} \mathrm{W}$, 3437 m, Yungas cloud forest, 23 Dec. 2009, AF 16348 \& PR (KRAM); by Sillu Tincara pre-Columbian route, $16^{\circ} 16^{\prime} 33^{\prime \prime} \mathrm{S}, 67^{\circ} 52^{\prime} 60^{\prime \prime} \mathrm{W}, 3429 \mathrm{~m}$, Yungas cloud forest, 27 June 2010, AF 16884, 16905, 16963 \& PR (KRAM, LPB).

Lepraria arbuscula (Nyl.) Lendemer \& Hodkinson

Leprocaulon arbuscula (Nyl.) Nyl.

In Bolivia known previously from only two records (Flakus \& Wilk 2006; Flakus 2008).

Specimens examined: BOliVia. Dept. La Paz. Prov. Nor Yungas, Cotapata a 3 horas caminando desde la comunidad de Yucupi, UTM 6248718210467 , $1705 \mathrm{~m}$, bosque no intervenido muy denso, corticolous, 7 Dec. 2006, PR \& S. Gallegos 322 (B, LPB); Hacienda Sacramento, ca $8 \mathrm{~km}$ del camino principal de Chuspipata hacia Coroico, $2500 \mathrm{~m}, 16^{\circ} 24^{\prime} \mathrm{S}, 67^{\circ} 47^{\prime} \mathrm{W}$, bosque siempreverde, epiphyte, 20 Sept. 1997, KB et al. 555 (B, LPB);

\section{Lepraria ecorticata (J. R. Laundon) Kukwa}

In Bolivia known previously from only three localities (Flakus \& Kukwa 2007; Flakus et al. 2011a).

Specimens examined: BOliVia. Dept. La Paz. Prov. Murillo, La Paz, Avenida Busch, desde la Paza Villaroel hasta el monumento a Busch, $3700 \mathrm{~m}, 16^{\circ} 28.9^{\prime} \mathrm{S}$, $68^{\circ} 07.2^{\prime} \mathrm{W}$, en Pinus radiata, 30 June 2008, G. Yapu-Alcázar 128 \& 132 (B, LPB); La Paz, Cota-Cota, Campus
Universitario, $3582 \mathrm{~m}, 16^{\circ} 32.3^{\prime} \mathrm{S}, 68^{\circ} 04.1^{\prime} \mathrm{W}$, puna y vegetación altoandina, on Schinus molle, 8 Feb. 2002, A. Canseco 138 (B, LPB); La Paz, Laguna de Cota-Cota (calle 31), sector frente a la puerta principal, $3750 \mathrm{~m}$, $16^{\circ} 32.4^{\prime} \mathrm{S}, 68^{\circ} 04.0^{\prime} \mathrm{W}$, Puna y vegetación altoandina, on Populus balsamifera, 8 Feb. 2002, A. Canseco 119 (B, LPB); La Paz, Plaza Villaroel, hacia Villa Fátima, $3685 \mathrm{~m}, 16^{\circ} 28.9^{\prime} \mathrm{S}, 68^{\circ} 07.3^{\prime} \mathrm{W}$, Puna y vegetación altoandina, on P. balsamifera, 8 Feb. 2002, A. Canseco 104 (B, LPB); La Paz, Plaza Adela Zamudio, Zona de Sopocachi, $3608 \mathrm{~m}, 16^{\circ} 30.9^{\prime} \mathrm{S}, 68^{\circ} 07.6^{\prime} \mathrm{W}$, area verde, on Acacia dealbata. 28 June 2008, G. Yapu-Alcázar 105 (B, LPB); La Paz, Plaza del Scoutt, $3560 \mathrm{~m}, 16^{\circ} 30.1^{\prime} \mathrm{S}$, $68^{\circ} 06.9^{\prime} \mathrm{W}$, area verde recreacional, on $A$. retinoides, 7 July 2008, G. Yapu-Alcázar 288 (B, LPB); La Paz, Plaza Rotary Club La Paz, Zona de Sopocachi, 3625 m, $16^{\circ} 30.6^{\prime} \mathrm{S}, 68^{\circ} 07.7^{\prime} \mathrm{W}$, area verde, on Pinus patula, 28 June 2008, G. Yapu-Alcázar 89 (B, LPB); La Paz, Plaza Vásquez, $3711 \mathrm{~m}, 16^{\circ} 29.3^{\prime} \mathrm{S}, 68^{\circ} 07.4^{\prime} \mathrm{W}$, area verde deficiente, on A. dealbata, 2 July 2008, G. YapuAlcázar 190 (B, LPB); La Paz, Plaza Villarroel, sector de las gradas, hacia Villa Fátima, $3685 \mathrm{~m}, 16^{\circ} 28.9^{\prime} \mathrm{S}$, $68^{\circ} 07.3^{\prime} \mathrm{W}$, puna y vegetacion altoandina, on Populus balsamifera, 8 Feb. 2002, A. D. Canseco-Tarifa 104 (B, LPB).

Lepraria finkii (B. de Lesd.) R. C. Harris

Lepraria lobificans auct. non Nyl.

The most often reported member of the genus in Bolivia but still known from only a few localities (Flakus \& Kukwa 2007; Flakus et al. 2011a, b; Flakus et al. 2012a).

Specimens examined: BOLIVIA. Dept. La PaZ. Prov. Larecaja, near Pajonalvilaque, $15^{\circ} 28^{\prime} 02^{\prime \prime} \mathrm{S}$, $68^{\circ} 02^{\prime} 12^{\prime \prime} \mathrm{W}, 890 \mathrm{~m}$, Preandean Amazon forest, on bark, 24 Nov. 2011, MK 10388 (LPB, UGDA, as admixture to Dichosporidium nigrocinctum).

\section{Lepraria gracilescens (Nyl.) Lendemer} \& Hodkinson

Leprocaulon gracilescens (Nyl.) I. M. Lamb \& A. M. Ward

Recorded in Bolivia only by Flakus and Wilk (2006), Flakus (2008) and Flakus et al. (2012a).

Chemistry. Atranorin, fatty acids.

Specimens examined: BOliVia. Dept. LA Paz. Prov. Camacho, cerca de la comunidad de Ulla Ulla, $4739 \mathrm{~m}, 15^{\circ} 02.068^{\prime} \mathrm{S}, 69^{\circ} 11.878^{\prime} \mathrm{W}$, vegetación 
altoandina con suelo muy pedregoso, terricolous, 4 Dec. 2007, PR $270 \& 280$ (B, LPB); Dept. Oruro. Prov. Sajama, SAJAMA, Ladera de la Quebrada Kohiri, 4309 m, $18^{\circ} 07.119^{\prime} \mathrm{S}, 68^{\circ} 56.108^{\prime} \mathrm{W}$, bosque de Polylepis tarapacana en ladera con piedras grandes, on rock, 21 Nov. 2007, RIS et al. 4381 (B, LPB); ibid., 4406 m, $18^{\circ} 07.132^{\prime} \mathrm{S}, 68^{\circ} 56.088^{\prime} \mathrm{W}$, bosque de Polylepis tarapacana, on rock, 21 Nov. 2007, RIS et al. 4395 (B, LPB).

\section{Lepraria impossibilis Sipman}

Reported from Bolivia only by Flakus and Kukwa (2007) but the specimens referred to as L. impossibilis were obscurely lobate and perhaps represent a different species. The records presented here are the first with truly lobate thalli.

Chemistry. Pannaric acid-6-methylester and related substances, lecanoric acid.

Specimens examined: BOLIVIA. Dept. Tarija. Prov. Aniceto Arce, Papachacra, $21^{\circ} 41^{\prime} 54^{\prime \prime} \mathrm{S}, 64^{\circ} 29^{\prime} 28^{\prime \prime} \mathrm{W}$, $2056 \mathrm{~m}$, Tucumano-Boliviano montane forest, corticolous, 27 Nov. 2010, AF 19942, 19947, 20041, 20047 \& $J Q$ (KRAM, LPB).

\section{Lepraria incana (L.) Ach.}

So far known in Bolivia from only a few records (Flakus \& Kukwa 2007; Flakus et al. 2011a, b)

Chemistry. Divaricatic acid and zeorin.

Specimen examined: BOliVia. Dept. La Paz. Prov. Franz Tamayo, APOLOBAMBA, Socondori Chico near Ulla Ulla, $15^{\circ} 00^{\prime} 38^{\prime \prime} \mathrm{S}, 6^{\circ} 13^{\prime} 48^{\prime \prime} \mathrm{W}, 4479 \mathrm{~m}$, high Andean open vegetation, saxicolous, 4 July 2010, $A F$ $17459 \&$ \& $(\mathrm{LPB})$.

\section{Lepraria neglecta (Nyl.) Erichsen}

Lepraria borealis Lohtander \& Tønsberg, L. caesioalba (B. de Lesd.) J. R. Laundon

Reported from Bolivia as L. borealis and L. caesioalba (Flakus \& Kukwa 2007; Flakus et al. 2011a), which are currently treated as chemotypes within L. neglecta (Lendemer 2013).

The status of L. alpina (B. de Lesd.) Tretiach \& Baruffo in South America, which is also included in the enlarged concept of L. neglecta, is unclear (Flakus et al. 2011b).
Chemistry. Fumarprotocetraric acid complex or psoromic acid as main diagnostic depsidones.

SPECIMENS EXAMINED (with fumarptorocetraric acid). BOlivia. Dept. La Paz. Prov. Bautista Saavedra, APOLOBAMBA, near Villa Amarca, $15^{\circ} 16^{\prime} 47^{\prime \prime} \mathrm{S}$, $69^{\circ} 01^{\prime} 47^{\prime \prime} \mathrm{W}, 4643 \mathrm{~m}$, Puna Húmeda, on bryophytes, 3 July 2010, $A F 17412$ \& PR (KRAM, LPB).

SPECimens EXAmined (with psoromic acid). BOLIVIA. Dept. La Paz. Prov. Franz Tamayo, APOLO-

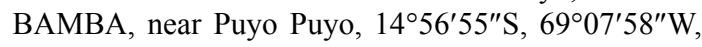
$4888 \mathrm{~m}$, high Andean open vegetation, on soil and bryophytes, 5 July 2010, AF 17583, $17650.1 \&$ \&R (KRAM, LPB); Prov. Murillo, near Cumbre pass, $16^{\circ} 21^{\prime} 59^{\prime \prime} \mathrm{S}$, $68^{\circ} 02^{\prime} 37^{\prime \prime} \mathrm{W}, 4604 \mathrm{~m}$, high Andean Puna, on soil and bryophytes, 20 Dec. 2009, AF 16292, 16318, $16319 \&$ \&R (KRAM, LPB); DePt. Oruro. Prov. Sajama, SAJAMA, Jecha K'ala, $18^{\circ} 09^{\prime} 52^{\prime \prime} \mathrm{S}, 68^{\circ} 49^{\prime} 08^{\prime \prime} \mathrm{W}, 4184 \mathrm{~m}$, Puna Sureña, pajonales vegetation, on soil and bryophytes, 20 June 2010, $A F$ 16602, 16613, 16667 \& PR (KRAM, LPB); near Sajama, $18^{\circ} 07^{\prime} 49^{\prime \prime} \mathrm{S}, 68^{\circ} 56^{\prime} 54^{\prime \prime} \mathrm{W}, 4437 \mathrm{~m}$, Puna Sureña, tholares vegetation, on soil and bryophytes, 18 June 2010, AF 16452, $16465 \&$ \&R (KRAM, LPB).

\section{Lepraria pallida Sipman}

In Bolivia known previously from only three localities (Flakus \& Kukwa 2007; Flakus et al. 2011a).

ChEMISTRY. Atranorin, zeorin and two unidentified fatty acids.

Specimens examined: BOliVia. Dept. La Paz. Prov. Omasuyos, Tajani cerca Achacachi, 15³6'49"S, $69^{\circ} 04^{\prime} 02^{\prime \prime} \mathrm{W}, 3869 \mathrm{~m}$, Puna Húmeda, on quartzite rock, 6 July 2010, AF 17795 \& PR (KRAM, LPB); DEPT. TARIJA. Prov. Aniceto Arce, Filo de Sidras, $22^{\circ} 14^{\prime} 50^{\prime \prime} \mathrm{S}$, $64^{\circ} 33^{\prime} 28^{\prime \prime} \mathrm{W}, 1064 \mathrm{~m}$, Tucumano-Boliviano submontane forest, corticolous, 22 Nov. 2010, AF 18693 (KRAM, LPB); Papachacra, 21 ${ }^{\circ} 41^{\prime} 54^{\prime \prime} \mathrm{S}, 64^{\circ} 29^{\prime} 28^{\prime \prime} \mathrm{W}$, $2056 \mathrm{~m}$, Tucumano-Boliviano montane forest, corticolous, 27 Nov. 2010, AF 19947.1 \& JQ (LPB).

\section{Lepraria rigidula (B. de Lesd.) Tønsberg}

This is the third record of this species for Bolivia (Flakus \& Kukwa 2007).

Chemistry. Atranorin and nephrosteranic acid.

Specimens examined: BOliVia. Dept. La Paz. Prov. Franz Tamayo, APOLOBAMBA, near Puyo Puyo, 
$14^{\circ} 56^{\prime} 55^{\prime \prime} \mathrm{S}, 6^{\circ} 07^{\prime} 58^{\prime \prime} \mathrm{W}, 4795 \mathrm{~m}$, high Andean open vegetation, on schist rock, 5 July 2010, AF 17659, 17662 $\& P R$ (KRAM, LPB).

\section{Lepraria vouauxii (Hue) R. C. Harris}

Probably one of the commonest Lepraria species in Bolivia (Flakus \& Kukwa 2007; Flakus et al. 2011a, b).

Chemistry. Pannaric acid-6-methylester and related substances.

Specimens examined: BOliVia. Dept. La Paz. Prov. Bautista Saavedra, APOLOBAMBA, near Taypi Cañuma, $15^{\circ} 03^{\prime} 20^{\prime \prime} \mathrm{S}, 69^{\circ} 09^{\prime} 07^{\prime \prime} \mathrm{W}, 4506 \mathrm{~m}$, high Andean open vegetation, on soil and bryophytes, 5 July 2010, AF 17510, 17522, 17532 \& PR (LPB); Prov. Franz Tamayo, APOLOBAMBA, near Puyo Puyo, $14^{\circ} 56^{\prime} 55^{\prime \prime} \mathrm{S}$, $69^{\circ} 07^{\prime} 58^{\prime \prime} \mathrm{W}, 4795-4888 \mathrm{~m}$, high Andean open vegetation, on soil and bryophytes, 5 July 2010, $A F$ 17625, 17650, 17652, 17669, $17691.1 \& P R$ (LPB); Prov. Murillo, near Cumbre pass, $16^{\circ} 21^{\prime} 59^{\prime \prime} \mathrm{S}, 68^{\circ} 02^{\prime} 37^{\prime \prime} \mathrm{W}$, $4604 \mathrm{~m}$, high Andean Puna, on soil and bryophytes, 20 Dec. 2009, $A F$ 16299, 16306, 16310, $16312 \& P R$ (KRAM, LPB); DePt. OruRo. Prov. Sajama, SAJAMA, Jecha K'ala, $18^{\circ} 09^{\prime} 52^{\prime \prime} \mathrm{S}, 68^{\circ} 49^{\prime} 08^{\prime \prime} \mathrm{W}, 4184 \mathrm{~m}$, Puna Sureña, pajonales vegetation, on soil, bryophytes or saxicolous, 20 June 2010, $A F$ 16617, 16650, 16676.2, $16680,16699 \&$ PR (KRAM, LPB); DePt. Potosí. Prov. Nor Lipez, Pinturas Rupestres near Mallku Villamar, $21^{\circ} 46^{\prime} 20^{\prime \prime} \mathrm{S}, 6^{\circ} 29^{\prime} 05^{\prime \prime} \mathrm{W}, 4038 \mathrm{~m}$, high Andean open semi-desert, on soil and bryophytes, 6 Dec. 2009, $A F$ $14805 \& P R$ (KRAM, LPB).

\section{Lepraria yunnaniana (Hue) Zahlbr.}

This is the fourth locality of this species in Bolivia (Kukwa \& Flakus 2009; Flakus et al. 2012a).

Chemistry. Divaricatic acid.

Specimens examined: BOliVia. Dept. La Paz. Prov. Nor Yungas, COTAPATA, by Sillu Tincara preColumbian route, $16^{\circ} 16^{\prime} 33^{\prime \prime} \mathrm{S}, 67^{\circ} 52^{\prime} 60^{\prime \prime} \mathrm{W}, 3429 \mathrm{~m}$, Yungas cloud forest, on bryophytes, 27 June 2010, $A F$ $16927 \& P R$ (KRAM, LPB).

\section{*Malmidea sulphureosorediata M. Cáceres,}

\section{A. Mota \& Aptroot}

Previously known only from the type locality in Brazil (Cáceres et al. 2013).
The thalli in the material examined were rather young and the soralia were still delimited.

Specimens examined: BOliVia. Dept. La Paz. Prov. Abel Iturralde, forest above Tumupasa village, $14^{\circ} 08^{\prime} 51^{\prime \prime} \mathrm{S}, 67^{\circ} 53^{\prime} 34^{\prime \prime} \mathrm{W}, 350 \mathrm{~m}$, Subandean Amazon forest, on bark of tree, 31 Aug. 2008, MK 7018a (LPB, UGDA);

Megalaria versicolor (Fée) Fryday \& Lendemer Lopezaria versicolor (Fée) Kalb \& Hafellner

This species, common in montane Yungas forest, has been reported from Bolivia only a few times (Flakus 2008; Flakus et al. 2012a).

Specimens eXamined: BOLIVIA. Dept. CochaBAMBA. Prov. Chapare, near Incachaca, $17^{\circ} 12^{\prime} 54^{\prime \prime} \mathrm{S}$, $65^{\circ} 49^{\prime} 30^{\prime \prime} \mathrm{W}, 2028 \mathrm{~m}$, Yungas cloud forest near road, corticolous, 7 July 2009, AF 12966 \& 12984 (LPB); CARRASCO, $123 \mathrm{~km}$ del camino antiguo de Cochabamba a Villa Tunari, $2100 \mathrm{~m}, 17^{\circ} 06^{\prime} \mathrm{S}, 65^{\circ} 37^{\prime} \mathrm{W}$, borde de camino, epiphyte, 10 Oct. 1997, KB et al. 746 \& 749 (LPB); $137 \mathrm{~km}$ del camino antiguo de Cochabamba a Villa Tunari, $1600 \mathrm{~m}, 17^{\circ} 06^{\prime} \mathrm{S}, 65^{\circ} 35^{\prime} \mathrm{W}$, bosque siempreverde, epiphyte, 11 Oct. 1997, KB et al. 766 (LPB); Dept. La Paz. Prov. Nor Yungas, entre Cotapata y Yucupi, UTM 6257458208812,1475 m, bosque intervenido cercano, sobre corteza, $4 \mathrm{Dec}$. 2006, PR 298a \& S. Gallegos (B, LPB); entre Cotapata y Yucupi, $1482 \mathrm{~m}, 16^{\circ} 11.1^{\prime} \mathrm{S}, 67^{\circ} 50.0^{\prime} \mathrm{W}$, bosque muy denso, corteza de árbol, 13 Maio 2006, PR et al. 99 (B, LPB); Cotapata, entre Sandillani y Bajo Hornuni, $1520 \mathrm{~m}, 16^{\circ} 11.1^{\prime} \mathrm{S}, 67^{\circ} 49.5^{\prime} \mathrm{W}$, bosque muy húmedo, corteza de árbol, 13 Oct. 2006, PR et al. $160 \& 165$ (B, LPB).

\section{Megalospora tuberculosa (Fée) Sipman}

In Bolivia known from only a few localities (Flakus et al. 2012a and literature cited therein).

Specimens examined: BOliVia. Dept. La Paz. Prov. Nor Yungas, Cotapata, 1 hora caminando desde Sandillani hacia Bajo Hornuni, $1520 \mathrm{~m}, 16^{\circ} 11.1^{\prime} \mathrm{S}$, $67^{\circ} 49.5^{\prime} \mathrm{W}$, bosque muy húmedo, corticolous, 13 Oct. 2006, PR, et al. 122, $163 \& 171$ (B, LPB); Prov. Sud Yungas, Alto Beni Sapecho, Colonia Tarapacá, $650 \mathrm{~m}$, $15^{\circ} 32^{\prime} \mathrm{S}, 67^{\circ} 21^{\prime} \mathrm{W}$, bosque siempreverde, epiphyte, 27 Apr. 1999, A. Acebey \& T. Krömer 980 (B, LPB); Alto Beni Sapecho, Conseción Cooperativa Sapecho, $650 \mathrm{~m}, 15^{\circ} 31^{\prime} \mathrm{S}, 67^{\circ} 20^{\prime} \mathrm{W}$, bosque siempreverde a semideciduo, epiphyte, 12 July 1999, A. Acebey \& T. Krömer 
976 (B, LPB); Dept. Santa Cruz. Prov. Florida, Refugio Los Volcanes, $1000 \mathrm{~m}, 18^{\circ} 06^{\prime} \mathrm{S}, 63^{\circ} 36^{\prime} \mathrm{W}$, bosque semi-siempreverde, epiphyte, 5 Oct. 1997, KB et al. 645 (LPB).

\section{${ }^{*}$ Parmelia warmingii Vain.}

Previously known only from Brazil (Vainio 1890; Zahlbruckner 1902; Hale \& Fletcher 1990; Spielmann 2009).

This poorly known species is characterized by its laciniate thallus with simple cilia up to $2.3 \mathrm{~mm}$ long, hypermaculate upper cortex, and the production of atranorin and salazinic acid (Spielmann 2009). It belongs to the taxonomically difficult Parmotrema cetratum group and was treated as a synonym of $P$. cetratum by Hale and Fletcher (1990) in their broad concept of this species. However, according to Spielmann (2009) it is a separate species, as $P$. cetratum differs from Parmelia warmingii by its laciniate thallus with distinct reticular maculae (not hypermaculate) and cilia up to $1.3 \mathrm{~mm}$ long. Its formal transfer to Parmotrema A. Massal. has not yet been made.

Chemistry. Atranorin, salazinic and consalazinic acids.

Specimens examined: BOliVia. Dept. La Paz. Prov. Larecaja, la Cumbre de Sorata, $15^{\circ} 53^{\prime} 06^{\prime \prime} \mathrm{S}$, $68^{\circ} 38^{\prime} 55^{\prime \prime} \mathrm{W}, 4787 \mathrm{~m}$, high Andean open vegetation, on soil, 13 May 2011, MK 8410 (LPB, UGDA); Prov. Nor Yungas, near Pongo village, near the road between

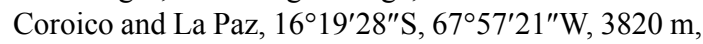
Páramo Yungueño, on rock, 25 May 2011, MK 9388 (LPB, UGDA).

\section{Parmotrema cetratum (Ach.) Hale}

In Bolivia known from only a few records; the older records may belong to other species (Flakus et al. 2012a).

Chemistry. Atranorin, salazinic and consalazinic acids.

Specimens examined: BOliVia. Dept. La Paz. Prov. Bautista Saavedra, on the road between Charazani and Apolo, $15^{\circ} 12^{\prime} 43^{\prime \prime} \mathrm{S}, 68^{\circ} 47^{\prime} 25^{\prime \prime} \mathrm{W}, 1650 \mathrm{~m}$, Yungas montane forest, open area with boulders, saxicolous, 18 May 2011, MK 8885 (LPB, UGDA).

\section{Parmotrema crocoides (Hale) Hale}

Reported in Bolivia from only three localities (Kukwa et al. 2012); the following is the first record from Santa Cruz Department.

Chemistry. Atranorin, gyrophoric acid, pigments, fatty acid; medulla patchily $\mathrm{C}+$ red just below cortex, pigmented parts $\mathrm{K}-, \mathrm{C}+$ orange.

Specimens examined: BOliVia. Dept. Santa Cruz. Prov. Ichilo, AMBORÓ, Macuñucu settlement, $17^{\circ} 43^{\prime} 38^{\prime \prime} \mathrm{S}, 63^{\circ} 35^{\prime} 37^{\prime \prime} \mathrm{W}, 460 \mathrm{~m}$, Amazon forest, on fallen branch, 6 June 2011, MK 9701 (LPB, UGDA).

\section{Parmotrema mellissii (C. W. Dodge) Hale}

In Bolivia known previously from only three localities, all in Cochabamba Department (Kukwa et al. 2012).

Chemistry. Atranorin, alectoronic acid, $\alpha$-collatolic acid and related substances; medulla $\mathrm{UV}+$ markedly bluish-white.

Specimens examined: BOliVia. Dept. La Paz. Prov. Nor Yungas, COTAPATA, Santa Catalina village, above Tunkini, $16^{\circ} 11^{\prime} 12^{\prime \prime} \mathrm{S}, 67^{\circ} 52^{\prime} 07^{\prime \prime} \mathrm{W}, 2700 \mathrm{~m}$, Yungas montane forest, on bark, 22 May 2011, MK 9024 (LPB); Dept. SANTA Cruz. Prov. Florida, AMBORÓ, above la Yunga village, senda Los Helechos, $18^{\circ} 03^{\prime} 30^{\prime \prime} \mathrm{S}, 63^{\circ} 54^{\prime} 36^{\prime \prime} \mathrm{W}, 2330 \mathrm{~m}$, Yungas cloud forest, corticolous, 7 June 2011, MK 9808 (LPB, UGDA).

\section{Phyllobaeis erythrella (Mont.) Kalb}

Only recently reported from Bolivia (Flakus et al. 2012a).

Specimens examined: BOLIVIA. Dept. La Paz. Prov. Bautista Saavedra, $3 \mathrm{~km}$ de Pauji-Yuyo hacia Charazani, $1100 \mathrm{~m}, 15^{\circ} 02^{\prime} \mathrm{S}, 68^{\circ} 29^{\prime} \mathrm{W}$, pared rocosa, on rock, 13 June 1997, KB et al. 156 (B, LPB).

\section{Phyllobaeis imbricata (Hook.) Kalb \& Gierl}

Reported from Bolivia several times (Flakus et al. 2012a and literature cited therein) but still under-recorded.

Specimens examined: BOlivia. Dept. CochaBAmba. Prov. Chapare, CARRASCO, $130 \mathrm{~km}$ del camino antiguo de Cochabamba a Villa Tunari, $2200 \mathrm{~m}$, $17^{\circ} 07^{\prime} \mathrm{S}, 65^{\circ} 36^{\prime} \mathrm{W}$, bosque húmedo con paredes rocosas, 
on rock, 10 Oct. 1997, KB et al. 681 (B, LPB); DEPT. LA PAz. Prov. Nor Yungas, COTAPATA, by Sillu Tincara pre-Columbian route, $16^{\circ} 16^{\prime} 33^{\prime \prime} \mathrm{S}, 67^{\circ} 52^{\prime} 60^{\prime \prime} \mathrm{W}$, $3429 \mathrm{~m}$, Yungas cloud forest, terricolous, 27 June 2010, $A F 16969 \&$ \&R (KRAM, LPB).

\section{Physcia undulata Moberg}

Reported from Bolivia only by Moberg (1990) and Canseco et al. (2006), from La Paz Department.

Specimens examined: BOLIVIA. Dept. Cochaвамва. Prov. Cercado, Cochabamba, $17^{\circ} 28.2^{\prime} \mathrm{S}$, $66^{\circ} 08.6^{\prime} \mathrm{W}, 2500 \mathrm{~m}$, vegetación de los valles secos interandinos, corticolous, 27 Sept. 2006, M. Canazas $M C-76$ (B, BOLV); ibidem, $17^{\circ} 22.9^{\prime} \mathrm{S}, 66^{\circ} 08.8^{\prime} \mathrm{W}$, 27 Sept. 2006, M.Canazas MC-79 (B, BOLV); DePt. La Paz. Prov. Murillo, La Paz, Plaza Lira, Zona de Sopocachi, $16^{\circ} 30.6^{\prime} \mathrm{S}, 68^{\circ} 08.0^{\prime} \mathrm{W}, 3687 \mathrm{~m}$, on Ulmus pumila, 28 June 2008, G. Yapu-Alcázar 85 (B, LPB); La Paz, Plaza Adela Zamudio, Zona de Sopocachi, $3608 \mathrm{~m}, 16^{\circ} 30,9^{\prime} \mathrm{S}, 68^{\circ} 07,6^{\prime} \mathrm{W}$, on Acacia melanoxylon and A. dealbata, 28 June 2008, G. Yapu-Alcázar $94 \& 97$ (B, LPB); La Paz, Avenida Busch, $3700 \mathrm{~m}, 16^{\circ} 28.9^{\prime} \mathrm{S}$, $68^{\circ} 07.2^{\prime} \mathrm{W}$, on Pinus radiata, 30 June 2008, G. YapuAlcázar 120 (B, LPB).

\section{Piccolia conspersa (Fée) Hafellner}

Reported in Bolivia from only four localities (Nylander 1859; Wilk 2010; Flakus et al. 2012a).

Specimens eXamined: BOliVia. Dept. CochaBAMBA. Prov. Chapare, CARRASCO, near Incachaca, $17^{\circ} 15^{\prime} 10^{\prime \prime} \mathrm{S}, 65^{\circ} 48^{\prime} 51^{\prime \prime} \mathrm{W}, 2330 \mathrm{~m}$, disturbed montane cloud forest, on bark of tree, 23 July 2008, MK 6737 (LPB, UGDA); Dept. La Paz. Prov. Nor Yungas, between Cotapata and Yucup, UTM 6257458208812 , $1475 \mathrm{~m}$, mountain forest, on bark, 4 Dec. 2006, PR 304 \& S. Gallegos (B, LPB); Dept. Tarija. Prov. Burnet $\mathrm{O}^{\prime}$ Connor, Lomas de la Soledad, road between Entre Ríos and Chiquiacá, $21^{\circ} 39^{\prime} 38^{\prime \prime} \mathrm{S}, 64^{\circ} 07^{\prime} 31^{\prime \prime} \mathrm{W}$, $1670 \mathrm{~m}$, Tucumano-Boliviano altimontano forest, on bark, 10 Aug. 2012, MK 11265 (LPB, UGDA).

\section{Placomaronea candelarioides Räsänen}

Reported in Bolivia from only two localities (Westberg et al. 2009).

Specimens examined: BOliVia. Dept. La Paz. Prov. Murillo, Camino a la Cumbre, $4250 \mathrm{~m}, \mathrm{ca} 16^{\circ} 0^{\prime} \mathrm{S}$, $68^{\circ} 0^{\prime} \mathrm{W}$, vegetación altoandino, saxicolous, 8 Feb. 2002, A. D. Canseco-Tarifa \& J. L. Vila 149 (B, LPB).

\section{Psiloparmelia distincta (Nyl.) Hale}

Reported only a few times from Bolivia (Nylander 1861; Hale 1989; Elix \& Nash 1992; Feuerer et al. 1998; Thell et al. 2004, 2006) but probably the commonest member of the genus in the country.

Chemistry. Atranorin, usnic, constipatic $( \pm)$ and protoconstipatic $( \pm)$ acids, and the fumarprotocetraric acid complex.

Specimens examined: BOliVia. Dept. La Paz. Prov. Bautista Saavedra, APOLOBAMBA, near Villa Amarca, $15^{\circ} 16^{\prime} 47^{\prime \prime} \mathrm{S}, 69^{\circ} 01^{\prime} 47^{\prime \prime} \mathrm{W}, 4643 \mathrm{~m}$, Puna Húmeda, saxicolous, 3 July 2010, AF 17360, 17384, $17429 \& P R$ (KRAM, LPB); near Taypi Cañuma, $15^{\circ} 03^{\prime} 20^{\prime \prime} \mathrm{S}, 69^{\circ} 09^{\prime} 07^{\prime \prime} \mathrm{W}, 4506 \mathrm{~m}$, high Andean open vegetation, saxicolous, 5 July 2010, $A F 17546 \& P R$ (KRAM, LPB); Prov. Camacho, Pacoamba cerca Wila Kala, $15^{\circ} 24^{\prime} 40^{\prime \prime} \mathrm{S}, 6^{\circ} 04^{\prime} 24^{\prime \prime} \mathrm{W}, 4283$ m, Puna Húmeda, saxicolous, 6 July 2010, $A F$ 17729, $17760 \&$ \&R (KRAM, LPB); Prov. Franz Tamayo, APOLOBAMBA, Socondori Chico near Ulla Ulla, $15^{\circ} 00^{\prime} 38^{\prime \prime} \mathrm{S}, 69^{\circ} 13^{\prime} 48^{\prime \prime} \mathrm{W}, 4479 \mathrm{~m}$, high Andean open vegetation, saxicolous, 4 July 2010, $A F$ 17466, 17488 \& PR (KRAM, LPB, herb. Flakus); Prov. Manco Kapac, near Copacabana Mt. Horca del Inca, $16^{\circ} 10^{\prime} 15^{\prime \prime} \mathrm{S}, 69^{\circ} 05^{\prime} 05^{\prime \prime} \mathrm{W}, 3974 \mathrm{~m}$, high Andean Puna, saxicolous, 18 June 2006, $A F$ 8616, 8626, 8635, \& 8665.1 (KRAM, LPB); Prov. Murillo, near Cumbre pass, $16^{\circ} 21^{\prime} 59^{\prime \prime} \mathrm{S}, 68^{\circ} 02^{\prime} 37^{\prime \prime} \mathrm{W}, 4604 \mathrm{~m}$, high Andean Puna, on schist rock, 13 May 2006, AF 5764, 16277, 16278, 16282, 16284, 16313, 16322 (KRAM, LPB, herb. Flakus); upper part of valley below Mt. Chacaltaya, $16^{\circ} 20^{\prime} 41^{\prime \prime} \mathrm{S}, 68^{\circ} 08^{\prime} 10^{\prime \prime} \mathrm{W}, 4980 \mathrm{~m}$, high Andean Puna, saxicolous, 9 Dec. 2004, $A F 4457$ (KRAM, LPB); Prov. Nor Yungas, near Pongo village, $16^{\circ} 19^{\prime} 28^{\prime \prime} \mathrm{S}$, $67^{\circ} 57^{\prime} 21^{\prime \prime} \mathrm{W}, 3820 \mathrm{~m}$, Páramo Yungueño, on rock, 25 May 2011, MK 9387 (LPB, UGDA); DePt. Oruro. Prov. Sajama, SAJAMA, Aguas Calientes near Sajama, $18^{\circ} 05^{\prime} 29^{\prime \prime} \mathrm{S}, 69^{\circ} 02^{\prime} 28^{\prime \prime} \mathrm{W}, 4448 \mathrm{~m}$, Puna Sureña, tholares vegetation, saxicolous, 18 June 2010, $A F 16518$, $16522,16531 \& P R$ (KRAM, LPB); Huincurata near Sajama, $18^{\circ} 07^{\prime} 01^{\prime \prime} \mathrm{S}, 68^{\circ} 58^{\prime} 00^{\prime \prime} \mathrm{W}, 4301 \mathrm{~m}$, Puna Sureña, Polylepis forest (Queñuales), saxicolous, 19 June 2010, $A F 16571 \& P R$ (KRAM, LPB); Jecha K'ala $25 \mathrm{~km}$ of Sajama, $18^{\circ} 09^{\prime} 52^{\prime \prime} \mathrm{S}, 68^{\circ} 49^{\prime} 08^{\prime \prime} \mathrm{W}, 4184 \mathrm{~m}$, Puna Sureña, pajonales vegetation, saxicolous, 20 June 2010, $A F$ $16615,16690 \& P R$ (LPB); near Sajama, $18^{\circ} 07^{\prime} 49^{\prime \prime} \mathrm{S}$, 
$68^{\circ} 56^{\prime} 54^{\prime \prime} \mathrm{W}, 4437 \mathrm{~m}$, Puna Sureña, tholares vegetation, saxicolous, 18 June 2010, $A F$ 16451, $16463 \& P R$ (KRAM, LPB, herb. Flakus).

\section{Punctelia stictica (Delise ex Duby) Krog}

Reported several times from Bolivia (Feuerer et al. 1998; Flakus et al. 2011b, 2012a) but underrecorded.

Specimens examined: BOliVia. Dept. La Paz. Prov. Murillo, above Yanacachi, $3000 \mathrm{~m}$, on rock, 6 Apr. 1987, S. Stab LB-66 (B); below Aldea Taquesi, 3800 m, on rock, 6 Apr. 1987, S. Stab LB-55 (B); Prov. Nor Yungas, Valle Unduavi, Huallara, $4100 \mathrm{~m}$, on rock, 28 May 1988, S. Stab LB-121 (B).

Ramalina asahinae W. Culb. \& C. Culb.

In Bolivia known previously from a single record (Feuerer \& Sipman 2005).

Chemistry. Boninic (major), 2-O-methylsekikaic (submajor) and 4'-O-methylpaludosic (trace) acids, usnic acid $( \pm)$.

Specimens examined: BOliVia. Dept. Santa Cruz. Prov. Florida, Amboró, below campamento in la Yunga village, $18^{\circ} 05^{\prime} 59^{\prime \prime} \mathrm{S}, 63^{\circ} 56^{\prime} 55^{\prime \prime} \mathrm{W}, 1648 \mathrm{~m}$, transition Yungas-Chiquitano forest, on bark, 8 June 2011, AF 22572 \& OP (KRAM, LPB, UGDA); ibid., near river, $18^{\circ} 06^{\prime} 40^{\prime \prime} \mathrm{S}, 63^{\circ} 56^{\prime} 59^{\prime \prime} \mathrm{W}, 1400 \mathrm{~m}$, transition Yungas-Chiquitano forests, on bark, 8 June 2011, $A F$ $22563 \& O P$ (KRAM, LPB, UGDA).

\section{*Ramalina aspera Räsänen}

Currently known from East Africa and in the Neotropics from Brazil, Colombia (see specimen below), Ecuador (the Galapagos), Guatemala, Mexico, Paraguay and Venezuela (Krog \& Swinscow 1976; López-Figueiras 1986; Kashiwadani \& Kalb 1993; Sipman \& Wolf 1998; Aptroot \& Bungartz 2007; Boom et al. 2007).

According to Kashiwadani and Kalb (1993) the species has two chemotypes, one with usnic and divaricatic acids and the other with usnic and cryptochlorophaeic acids. Our specimens contained boninic acid with related substances, never reported from this species before, but our material agrees with the protologue and is morphologically identical with material revised by Kashiwadani (deposited in BM), and therefore it is referred to R. aspera.

Chemistry. Boninic (major or submajor), 2-O-methylsekikaic (submajor or major), 4'-Omethylpaludosic (submajor or trace) and 4,4'-diO-methylcryptochlorophaeic acid ( \pm , trace) acids.

Specimens examined: BOliVia. Dept. Santa Cruz. Prov. Cordillera, KAA-IYA, Charrata, $18^{\circ} 28^{\prime} 05^{\prime \prime}$, $62^{\circ} 05^{\prime} 43^{\prime \prime} \mathrm{W}, 307 \mathrm{~m}$, Chaqueño forest, on bark, 2 Dec. 2010, $A F 20117$ \& JQ (KRAM, LPB); DePT. TARIJA. Prov. Aniceto Arce, Papachacra, 21 ${ }^{\circ} 41^{\prime} 36^{\prime \prime} \mathrm{S}$, $64^{\circ} 29^{\prime} 33^{\prime \prime} \mathrm{W}, 2195 \mathrm{~m}$, Tucumano-Boliviano montane forest, on bark, 26 Nov. 2010, $A F 19787 \& J Q$ (LPB).

Comparative SPECIMEN EXAMINED: COLOMBIA. CALI. River Panse, $1000 \mathrm{~m}, 3^{\circ} 28^{\prime} \mathrm{N}, 76^{\circ} 30^{\prime \prime} \mathrm{W}$, on tree, 30 Oct. 1967, R. M. Garrett 21 (BM; cryptochlorophaeic acid strain).

${ }^{*}$ Ramalina canaguensis V. Marcano \& A. Morales

Previously known only from a few records in Colombia and Venezuela (Marcano \& Morales 1994; Sipman 2006).

Chemistry. Usnic acid.

Specimens examined: BOliVia. Dept. Santa Cruz. Prov. Manuel María Caballero, near Siberia, $17^{\circ} 49^{\prime} 38^{\prime \prime} \mathrm{S}, 64^{\circ} 44^{\prime} 45^{\prime \prime} \mathrm{W}, 3950 \mathrm{~m}$, Yungas cloud forest, on bark, 16 Aug. 2012, MK 11449 (LPB, UGDA).

\section{Ramalina celastri (Spreng.) Krog \& Swinscow}

Reported several times from Bolivia (Nylander 1859, 1861; Herzog 1922; Feuerer \& Sipman 2005; Flakus 2008; Flakus et al. 2012a), but underrecorded in the country. Here it is reported for the first time from Tarija Department.

\section{Chemistry. Usnic acid ( \pm ).}

Specimens examined: BOLIVIA. Dept. La PaZ. Prov. Larecaja, Titilaya, $15^{\circ} 39^{\prime} 55^{\prime \prime} \mathrm{S}, 68^{\circ} 42^{\prime} 51^{\prime \prime} \mathrm{W}$, $2773 \mathrm{~m}$, transition Yungas forest-Valles Secos vegetation, on bark, 14 May 2011, $A F 20357$ \& OP (LPB); DePt. SANTA Cruz. Prov. Cordillera, KAA-IYA, near Peto Blanco, $18^{\circ} 51^{\prime} 36^{\prime \prime} \mathrm{S}, 60^{\circ} 18^{\prime} 00^{\prime \prime} \mathrm{W}, 290 \mathrm{~m}$, transition Chiquitano-Chaqueño forest, on bark, 5 Dec. 2011, AF 23726 (KRAM, LPB); Dept. TARIJA. Prov. Aniceto Arce, Filo de Sidras, $22^{\circ} 14^{\prime} 50^{\prime \prime} \mathrm{S}, 64^{\circ} 33^{\prime} 28^{\prime \prime} \mathrm{W}$, $1064 \mathrm{~m}$, Tucumano-Boliviano submontane forest, on 
bark, 22 Nov. 2010, AF 18474.1, 18523.2, 18588, 18599, 18634 (KRAM, LPB); Papachacra, 21 ${ }^{\circ} 41^{\prime} 52^{\prime \prime}$, $64^{\circ} 29^{\prime} 15^{\prime \prime} \mathrm{W}, 1900 \mathrm{~m}$, Tucumano-Boliviano altimontane forest, on bark, 8 Aug. 2012, AF 23975 (KRAM, LPB); Papachacra, 21 ${ }^{\circ} 41^{\prime} 36^{\prime \prime} \mathrm{S}, 64^{\circ} 29^{\prime} 33^{\prime \prime} \mathrm{W}, 2195 \mathrm{~m}$, Tucumano-Boliviano montane forest, on bark, 26 Nov. 2010, AF $19902 \& J Q$ (LPB).

\section{Ramalina cochlearis Zahlbr.}

In Bolivia known previously from only one locality (Flakus et al. 2013).

CHEMISTRY. Usnic acid, homosekikaic (major) and sekikaic (minor) acids.

Specimens examined: BOlivia. Dept. La Paz. Prov. Nor Yungas, Coroico, $16^{\circ} 11^{\prime} 10^{\prime \prime} \mathrm{S}, 67^{\circ} 43^{\prime} 16^{\prime \prime} \mathrm{W}$, $1550 \mathrm{~m}$, Yungas montane forest, on bark, 6 June 2010, $A F 16415.1 \& P R$ (KRAM, LPB); Coroico, near Sol y Luna hotel, $1870 \mathrm{~m}, 16^{\circ} 11^{\prime} 56^{\prime \prime} \mathrm{S}, 67^{\circ} 43^{\prime} 26^{\prime \prime} \mathrm{W}$, gardens with trees, on bark, 13 Dec. 2011, MK 10741 (LPB).

\section{*Ramalina disparata Krog \& Swinscow}

Previously reported from Peru in South America and elsewhere from Africa and Australia (Krog \& Swinscow 1976; Kashiwadani 1987; McCarthy 2014).

Chemistry. Usnic acid $( \pm)$, boninic (major), 2-O-methylsekikaic (major) and 4'-O-methylpaludosic (minor) acids.

Specimens examined: BOliVia. Dept. CochaBAmba. Prov. Carrasco, CARRASCO, near Sehuencas village, $17^{\circ} 30^{\prime} 12^{\prime \prime} \mathrm{S}, 6^{\circ} 16^{\prime} 30^{\prime \prime} \mathrm{W}, 2220 \mathrm{~m}$, Yungas montane cloud forest, on bark of tree, 21 July 2008, $M K$ 6454 (LPB, UGDA); Prov. Chapare, CARRASCO, near Incachaca, $17^{\circ} 15^{\prime} 10^{\prime \prime} \mathrm{S}, 65^{\circ} 48^{\prime} 51^{\prime \prime} \mathrm{W}, 2330 \mathrm{~m}$, Yungas montane cloud forest, on bark of tree, 23 July 2008, $M K$ 6739 (LPB, UGDA); DePt. La Paz. Prov. Nor Yungas, Coroico, near Sol y Luna hotel, $1870 \mathrm{~m}, 16^{\circ} 11^{\prime} 56^{\prime \prime} \mathrm{S}$, $67^{\circ} 43^{\prime} 26^{\prime \prime} \mathrm{W}$, gardens with trees, on bark, 13 Dec. 2011, MK 10793 (LPB, UGDA); DePt. TARIJA. Prov. Aniceto Arce, El Carmen, $21^{\circ} 58^{\prime} 50^{\prime \prime} \mathrm{S}, 64^{\circ} 37^{\prime} 31^{\prime \prime} \mathrm{W}, 2700 \mathrm{~m}$, open semi-desert vegetation with small shrubs, on bark, 9 Aug. 2012, MK 11128a (LPB, UGDA).

\section{${ }^{*}$ Ramalina grumosa Kashiw.}

Previously known only from Brazil and Peru (Kashiwadani 1987; Kashiwadani \& Kalb 1993).
Chemistry. Usnic acid ( \pm , minor), boninic (major to minor), 2-O-methylsekikaic (submajor to trace), 4'-O-methylpaludosic ( \pm , trace), salazinic $( \pm$, major) and protocetraric ( \pm , trace) acids.

Specimens examined: BOLIVIA. Dept. La Paz. Prov. Nor Yungas, Coroico, $16^{\circ} 11^{\prime} 10^{\prime \prime} \mathrm{S}, 67^{\circ} 43^{\prime} 16^{\prime \prime} \mathrm{W}$, $1550 \mathrm{~m}$, Yungas montane forest, on bark, 6 June 2010, AF $16415 \&$ \&R (KRAM, LPB); Dept. TARIJA. Prov.

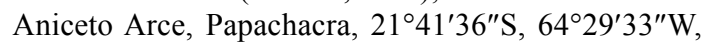
$2195 \mathrm{~m}$, Tucumano-Boliviano montane forest, on bark, 26 Nov. 2010, AF 19894 \& $J Q$ (LPB).

\section{${ }^{*}$ Ramalina incana Kashiw.}

Previously known only from Peru (Kashiwadani 1987).

\section{Chemistry. Usnic acid.}

Specimens examined: BOliVia. Dept. La Paz. Prov. Omasuyos, El Dragon hill near Chahualla, $15^{\circ} 51^{\prime} 17^{\prime \prime} \mathrm{S}, 69^{\circ} 00^{\prime} 40^{\prime \prime} \mathrm{W}, 3850 \mathrm{~m}$, Puna Húmeda, on rock, 6 July 2010, $A F 17813 \&$ PR (KRAM, LPB, UGDA).

\section{Ramalina peruviana Ach.}

Rarely reported in Bolivia (Flakus et al. 2013).

Chemistry. Usnic acid $( \pm)$, homosekikaic (major) and sekikaic (minor) acids.

Specimens examined: BOliVia. Dept. TariJa. Prov. Aniceto Arce, SSE of Padcaya, $22^{\circ} 14^{\prime} 56^{\prime \prime} \mathrm{S}$, $64^{\circ} 32^{\prime} 55^{\prime \prime} \mathrm{W}, 883 \mathrm{~m}$, Tucumano-Boliviano secondary submontane forest, on bark, 21 Nov. 2010, AF 18365.1 (LPB); Prov. Burnet O'Connor, TARIQUÍA, near Salinas, $21^{\circ} 49^{\prime} 15^{\prime \prime} \mathrm{S}, 64^{\circ} 12^{\prime} 44^{\prime \prime} \mathrm{W}, 1400 \mathrm{~m}$, TucumanoBoliviano montane forest, corticolous, 10 Aug. 2012, $A F \& M K 11220$ (LPB, UGDA).

\section{${ }^{*}$ Ramalina puiggarii Müll. Arg.}

Previously known only from Brazil and Ecuador (the Galapagos) (Kashiwadani \& Kalb 1993; Aptroot \& Bungartz 2007).

\section{Chemistry. Usnic acid.}

Specimens examined: BOliVia. Dept. La Paz. Prov. Nor Yungas, COTAPATA, by Sillu Tincara pre-Columbian route, $16^{\circ} 17^{\prime} 22^{\prime \prime} \mathrm{S}, 67^{\circ} 53^{\prime} 29^{\prime \prime} \mathrm{W}, 3518 \mathrm{~m}$, transition Páramo Yungueño-Yungas cloud forest, 25 May 2011, on bark, $A F 21795$ \& OP (KRAM, LPB). 


\section{${ }^{*}$ Ramalina usnea (L.) Howe}

Previously reported from Argentina, Brazil, Chile, Colombia, Ecuador (Galapagos), Venezuela and North America (López-Figueiras 1986; Kashiwadani \& Kalb 1993; Galloway \& Quilhot 1998; Calvelo \& Liberatore 2002; Aptroot \& Bungartz 2007; Sipman et al. 2008; Esslinger 2014).

ChEMISTRY. Homosekikaic (major) and sekikaic (submajor) acids, traces of unknown substances.

Specimens examined: BOliVia. Dept. La Paz. Prov. Dept. Santa Cruz. Prov. Cordillera, KAA-IYA, near Peto Blanco, $18^{\circ} 51^{\prime} 36^{\prime \prime} \mathrm{S}, 60^{\circ} 18^{\prime} 00^{\prime \prime} \mathrm{W}, 290 \mathrm{~m}$, transition Chiquitano-Chaqueño forest, on bark, 6 Dec. 2011, $A F 23721$ (KRAM, LPB).

\section{${ }^{*}$ Ramonia valenzueliana (Mont.) Stizenb.}

Previously reported from Brazil, Cuba, Ecuador (Galapagos), Paraguay and the USA (Florida \& Louisiana) (Vězda 1966; Tucker 1981; Bungartz et al. 2013; Esslinger 2014).

Specimens examined: BOliVia. Dept. Santa CRuz Prov. Cordillera, between Tucavaca and Roboré, $18^{\circ} 37^{\prime} 56^{\prime \prime} \mathrm{S}, 59^{\circ} 36^{\prime} 50^{\prime \prime} \mathrm{W}, 370 \mathrm{~m}$, transition ChiquitanoChaqueño forest, 4 Dec. 2011, AF 23418 (LPB, KRAM).

\section{Relicina abstrusa (Vain.) Hale}

This is the third record of this species from Bolivia (Flakus et al. 2011b, 2012a).

Chemistry. Usnic, norstictic, connorstictic and hyposalazinic acids.

Specimens eXamined: BOLIVIA. Dept. Tarija. Prov. Burnet O'Connor, Lomas de la Soledad, road between Entre Ríos and Chiquiacá, $21^{\circ} 39^{\prime} 38^{\prime \prime} \mathrm{S}, 64^{\circ} 07^{\prime} 31^{\prime \prime} \mathrm{W}$, $1670 \mathrm{~m}$, Tucumano-Boliviano forest, on rock, 10 Aug. 2012, MK11257 (LPB, UGDA).

\section{Remototrachyna costaricensis (Nyl.) Divakar \& A. Crespo}

Reported from several localities in Bolivia (see Flakus et al. 2011b, 2012a) but under-recorded.

Chemistry. Atranorin and consitpatic acid complex.

Specimen examined: BOlivia. Dept. La Paz.
Prov. Bautista Saavedra, between Apolo and Charazani, $15^{\circ} 11^{\prime} 02^{\prime \prime} \mathrm{S}, 68^{\circ} 53^{\prime} 43^{\prime \prime} \mathrm{W}, 2560 \mathrm{~m}$, transition Yungas montane cloud forest, on rock, 18 May 2011, MK 8938 (LPB).

Remototrachyna sipmaniana Kukwa \& Flakus

Recently described from two localities in Bolivia (Flakus et al. 2012b). The new record is close to the type locality.

Chemistry. Atranorin, protocetraric and gyrophoric acids (the latter substance only in isidia, which are $\mathrm{C}+$ red).

Specimens examined: BOliVia. Dept. CochaBAMBA. Prov. Carrasco, CARRASCO, near Sehuencas, $17^{\circ} 26^{\prime} 14^{\prime \prime} \mathrm{S}, 65^{\circ} 15^{\prime} 20^{\prime \prime} \mathrm{W}, 2150 \mathrm{~m}$, Yungas cloud forest, on rock, 18 Aug. 2012, MK 11743 (LPB, UGDA).

Siphula fastigiata (Nyl.) Nyl.

Only three previous records from Bolivia (Kantvilas 1998; Kantvilas \& Elix 2002; Flakus et al. 2014).

Chemistry. Baeomycetic and squamatic acids.

Specimens examined: BOliVia. Dept. La Paz. Prov. Larecaja, la Cumbre de Sorata, $15^{\circ} 53^{\prime} 06^{\prime \prime} \mathrm{S}$, $68^{\circ} 38^{\prime} 55^{\prime \prime} \mathrm{W}, 4787 \mathrm{~m}$, high Andean open vegetation, on soil, 13 May 2011, MK 8393 (LPB, UGDA); Prov. Murillo, Chacaltaya, $16^{\circ} 21^{\prime} 12^{\prime \prime} \mathrm{S}, 68^{\circ} 07^{\prime} 53^{\prime \prime} \mathrm{W}, 4700$ $4900 \mathrm{~m}$, on ground, 1983, C. Halls 1984/84 (BM); Prov. Nor Yungas, near Pongo, $16^{\circ} 19^{\prime} 28^{\prime \prime} \mathrm{S}, 67^{\circ} 57^{\prime} 21^{\prime \prime} \mathrm{W}$, 3820 m, Páramo Yungueño, 25 May 2011, MK 9394 (LPB, UGDA).

Stereocaulon alpestre (Flot.) Dombr.

Stereocaulon tomentosum Th. Fr. var. alpestre Flot.

The only previous record of this species from Bolivia is by Herzog (1922).

Specimens examined: BOliVia. Dept. CochaBAmba. Prov. Ayopaya, Saila Pata, $3500 \mathrm{~m}, 16^{\circ} 55^{\prime} \mathrm{S}$, $66^{\circ} 55^{\prime} \mathrm{W}$, in forest, saxicolous, 15 Nov. $1997, K B \& I$. Jimenez 910 (B, LPB).

Stereocaulon glareosum (Sav.) H. Magn.

Previously reported from Bolivia by Sipman (1992, 2002), Feuerer et al. (1998) and Flakus et al. (2011b). 
Chemistry. Atranorin and perlatolic acid.

Specimens examined: BOliVia. Dept. La Paz. Prov. Sud Yungas, Mururata, $4000 \mathrm{~m}, 16^{\circ} 35^{\prime} \mathrm{S}, 67^{\circ} 45^{\prime} \mathrm{W}$, pastizal semialto, saxicolous, 1995, J. Gonzáles 1499 (B, LPB).

\section{Stereocaulon meyeri Stein}

Previously reported from Boliva by Lamb (1977), Feuerer et al. (1998), Oset and Kukwa (2012) and Flakus et al. (2012a).

Specimens examined: BOliVia. Dept. La Paz. Prov. Camacho, cerca de la Estancia Taypi Kañuma, en el cerro Pelechucomita, $5050 \mathrm{~m}, 15^{\circ} 01.432^{\prime} \mathrm{S}$, $69^{\circ} 7.917^{\prime} \mathrm{W}$, vegetación altoandina, terricolous, $4 \mathrm{Dec}$. 2008, RIM 4469 (B, LPB).

Stereocaulon myriocarpum Th. Fr.

This is the third record of this species from Bolivia (Flakus et al. 2012a).

Specimens examined: BOliVia. Dept. La Paz. Prov. Sud Yungas, Camino hacia el Illimani, $4010 \mathrm{~m}$, $16^{\circ} 45^{\prime} \mathrm{S}, 67^{\circ} 45^{\prime} \mathrm{W}$, pastizal semialto, saxicolous, $26 \mathrm{Feb}$. 1995, J. Gonzáles 181 (B, LPB).

\section{Stereocaulon pityrizans Nyl.}

Noted from Bolivia by Sipman (2002) and Flakus et al. (2011b, 2012a).

Specimens examined: BOLIVIA. Dept. La Paz. Prov. Nor Yungas, $5 \mathrm{~km}$ del camino principal de Chuspipata hacia Coroico, $2700 \mathrm{~m}, 16^{\circ} 23^{\prime} \mathrm{S}, 67^{\circ} 48^{\prime} \mathrm{W}$, pared rocosa en el borde de camino, terricolous, 19 Sept. 1997, $K B$ et al. 550 (B, LPB).

\section{Stereocaulon ramulosum (Sw.) Räusch}

The commonest member of the genus in Bolivia (Rodriguez et al. 2014), but restricted to montaine forests.

Specimens examined: BOliVia. Dept. CochaBAmba. Prov. Ayopaya, $10 \mathrm{~km}$ de Cocapata hacia Cotacajes, $2700 \mathrm{~m}, 16^{\circ} 38^{\prime} \mathrm{S}, 66^{\circ} 41^{\prime} \mathrm{W}$, bosque húmedo, on wood, 11 May 1997, KB et al. 27 (B, LPB); comunidad de Saila Pata, $3500 \mathrm{~m}, 16^{\circ} 55^{\prime} \mathrm{S}, 66^{\circ} 55^{\prime} \mathrm{W}$, sendero con rocas en bosque siempreverde, on rock, 15 Nov. 1997, KB \& I. Jimenez 908 (B, LPB); Prov. Chapare, CARRASCO, $130 \mathrm{~km}$ del camino antiguo de Cochabamba a Villa Tunari, $2200 \mathrm{~m}, 17^{\circ} 07^{\prime} \mathrm{S}, 65^{\circ} 36^{\prime} \mathrm{W}$, bosque húmedo, on rock, 10 Oct. 1997, KB et al. 684 (B, LPB); DEPT. LA PAz. Prov. Bautista Saavedra, $15 \mathrm{~km}$ de Charazani hacia Apolo, $2400 \mathrm{~m}, 15^{\circ} 11^{\prime} \mathrm{S}, 68^{\circ} 52^{\prime} \mathrm{W}$, bosque húmedo secundario, on rocks, $1 \& 2$ July $1997, K B$ et al. $275 \& 298$ (LPB); Prov. Nor Yungas, $2 \mathrm{~km}$ del camino principal de Chuspipata hacia Coroico, $2900 \mathrm{~m}, 16^{\circ} 22^{\prime} \mathrm{S}, 67^{\circ} 49^{\prime} \mathrm{W}$, el borde de camino, on rock, 17 Sept. 1997, KB et al. 541 (B, LPB); Coscapa, senda al oueste del camino principal $c a$ $4 \mathrm{~km}$ de Cotapata hacia La Paz, 3300-3450 m, 16 $23^{\prime} \mathrm{S}$, $67^{\circ} 53^{\prime} \mathrm{W}$, pastizal, on rock, $9 \& 10$ Sept. 1997, KB et al. 472, 473, 480 \& 490 (LPB); Serranía Bellavista, ca $36 \mathrm{~km}$ por camino de Caranavi hacia Sapecho, $1500 \mathrm{~m}, 15^{\circ} 41^{\prime} \mathrm{S}$, $67^{\circ} 30^{\prime} \mathrm{W}$, on rock, 31 Aug. 1997, KB et al. 434 (B, LPB); Serranía Bellavista, $c a 43 \mathrm{~km}$ por camino de Caranavi hacia Sapecho. $1400 \mathrm{~m}, 15^{\circ} 41^{\prime} \mathrm{S}, 67^{\circ} 30^{\prime} \mathrm{W}$, borde del camino, on rock, 27 Aug. 1997, KB et al. 426 (B, LPB); Prov. Sud Yungas, Illimani, camino hacia la Mina Bolsa Blanca, $4000 \mathrm{~m}, 16^{\circ} 45^{\prime} \mathrm{S}, 67^{\circ} 45^{\prime} \mathrm{W}$, on rock, 11 Nov. 1995, J. Gonzáles 1395 (B, LPB); Mururata, bajo la Mina Bolsa Negra, pasando por el pueblo de Tres Rios, $4040 \mathrm{~m}, 16^{\circ} 35^{\prime} \mathrm{S}, 67^{\circ} 45^{\prime} \mathrm{W}$, pastizal semialto, on rock, 1995, J. Gonzáles 419, 1035, 1395 \& 2038 (B, LPB); ibid., $3900 \mathrm{~m}$, pastizal semialto, on rock, 9 Dec. 1994, J. Gonzáles 127 (B, LPB); ibid., 3935 m, on rock, 27 Feb. 1995, J. Gonzáles 224 (B, LPB).

\section{Stereocaulon strictum Th. Fr.}

Reported from Bolivia by Lamb (1977), Sipman (1992), Feuerer et al. (1998) and Flakus et al. (2011b, 2012a), with detailed locality data provided in the latter three works.

Specimens examined: BOLIVIA. Dept. CochaBAMBA. Prov. Chapare, CARRASCO, $132 \mathrm{~km}$ del camino antiguo de Cochabamba a Villa Tunari, $1950 \mathrm{~m}, 17^{\circ} 06^{\prime} \mathrm{S}$, $65^{\circ} 35^{\prime} \mathrm{W}$, borde de camino en bosque húmedo, on rock, 10 Oct. 1997, KB et al 674 (B, LPB, var. strictum); $130 \mathrm{~km}$ del camino antiguo de Cochabamba a Villa Tunari, $2200 \mathrm{~m}, 17^{\circ} 07^{\prime} \mathrm{S}, 65^{\circ} 36^{\prime} \mathrm{W}$, bosque húmedo, saxicolous, 10 Oct. 1997, KB et al. 703 (B, LPB, var. strictum); DePt. LA Paz. Prov. Nor Yungas, $2 \mathrm{~km} \mathrm{del}$ camino principal de Chuspipata hacia Coroico. $2900 \mathrm{~m}$, $16^{\circ} 22^{\prime} \mathrm{S}, 67^{\circ} 49^{\prime} \mathrm{W}$, pared rocosa en el borde de camino, saxicolous, 17 Sept. 1997, KB et al. 540 (B, LPB, var. compressum (Nyl.) Lamb ex Vězda).

\section{Stereocaulon vesuvianum Pers.}

Noted from Bolivia in a few papers (Sipman 2002; Feuerer \& Sipman 2005; Flakus et al. 2011b, 2012a) but still considered rare in the country. 
Specimens examined: BOliVia. Dept. La Paz. Prov. Sud Yungas, Mururata, bajo la Mina Bolsa Negra, $4000 \mathrm{~m}, 16^{\circ} 35^{\prime} \mathrm{S}, 67^{\circ} 45^{\prime} \mathrm{W}$, pastizal semialto, saxicolous, 1995, J. Gonzáles 1429 (B, LPB);

\section{Teloschistes exilis (Michx.) Vain.}

Previously known in Bolivia from a single recent record (Lynge 1925; Malme 1926; Flakus et al. 2012a).

SPeCimens eXamined: BOLIVIA. Dept. CochaBAmba. Prov. Ayopaya, $10 \mathrm{~km}$ de Cocapata hacia Cotacajes, $2800-2850 \mathrm{~m}, 16^{\circ} 38^{\prime} \mathrm{S}, 66^{\circ} 41^{\prime} \mathrm{W}$, bosque húmedo, on bark, $10 \& 12$ May 1997, KB et al. $9,45 \& 63$ (LPB); $20 \mathrm{~km}$ de Cocapata hacia Cotacajes, $2000 \mathrm{~m}, 16^{\circ} 46^{\prime} \mathrm{S}$, $66^{\circ} 44^{\prime} \mathrm{W}$, bosque semideciduo disturbado, on bark, 15 May 1997, KB et al. 80, 105 \& 124d (B, LPB); $5 \mathrm{~km}$ al sur de Saila Pata, $3050 \mathrm{~m}, 16^{\circ} 55^{\prime} \mathrm{S}, 66^{\circ} 56^{\prime} \mathrm{W}$, bosque siempreverde, on bark, 10 Nov. 1997, KB 865 \& 866 \& I. Jimenez (B, LPB); cancha de fútbol en Karhuani, $2950 \mathrm{~m}, 16^{\circ} 51^{\prime} \mathrm{S}, 66^{\circ} 54^{\prime} \mathrm{W}$, plantación con Eucalyptus, on bark, 8 Nov. 1997, KB 925 (B, LPB); comunidad de Saila Pata. $3200 \mathrm{~m}, 16^{\circ} 54^{\prime} \mathrm{S}, 66^{\circ} 56^{\prime} \mathrm{W}$, SW-exposicion, bosque siempreverde con Podocarpus, epiphyte, 12 Nov. 1997, KB 887, 887a \& 892a \& I. Jimenez (B, LPB); Dept. LA Paz. Prov. Bautista Saavedra, $10 \mathrm{~km}$ de Charazani hacia Apolo, $2550 \mathrm{~m}, 15^{\circ} 11^{\prime} \mathrm{S}, 68^{\circ} 52^{\prime} \mathrm{W}$, pared rocosa, 31 May 1997, KB et al. 125 (B, LPB); $15 \mathrm{~km}$ de Charazani hacia Apolo, $2400 \mathrm{~m}, 15^{\circ} 11^{\prime} \mathrm{S}$, $68^{\circ} 52^{\prime} \mathrm{W}$, pastos con arbustos al lado del Río Camata, terricolous, 1 July 1997, KB et al. 250 (LPB); Prov. Nor Yungas, San Vincente, $\mathrm{ca} 1 \mathrm{~km}$ al oeste de Chuspipata. $3000 \mathrm{~m}, 16^{\circ} 21^{\prime} \mathrm{S}, 67^{\circ} 49^{\prime} \mathrm{W}$, asfalto viejo por una cancha de fútbol con árbustos, on rock, 21 Sept. 1997, KB et al. 584 (LPB).

\section{Teloschistes flavicans (Sw.) Norman}

Reported from Bolivia several times but still under-recorded; few records are recent (Nylander 1859, 1861; Rusby 1896; Lynge 1925; Feuerer et al. 1998; Søchting \& Fröden 2002; Flakus et al. 2012a).

Specimens examined: BOliVia. Dept. CochaBAmba. Prov. Ayopaya, $10 \mathrm{~km}$ de Cocapata hacia Cotacajes, $2800-2850 \mathrm{~m}, 16^{\circ} 38^{\prime} \mathrm{S}, 66^{\circ} 41^{\prime} \mathrm{W}$, bosque húmedo, on bark, 10 May 1997, KB et al. $9 a$ (LPB); $20 \mathrm{~km}$ de Cocapata hacia Cotacajes, $2000 \mathrm{~m}, 16^{\circ} 46^{\prime} \mathrm{S}, 66^{\circ} 44^{\prime} \mathrm{W}$, bosque semideciduo disturbado, on bark, 17 May 1997, $K B$ et al. $124 a$ (B, LPB); $5 \mathrm{~km}$ al sur de Saila Pata. $3050 \mathrm{~m}, 16^{\circ} 55^{\prime} \mathrm{S}, 66^{\circ} 56^{\prime} \mathrm{W}$, bosque siempreverde, on bark, 10 Nov. 1997, KB 856 \& I. Jimenez (B, LPB); Dept. La Paz. Prov. Bautista Saavedra, $15 \mathrm{~km} \mathrm{de}$ Camata hacia Apolo, $1400 \mathrm{~m}, 15^{\circ} 13^{\prime} \mathrm{S}, 68^{\circ} 41^{\prime} \mathrm{W}$, bosque semi-deciduo, on bark, 27 June 1997, KB et al. 223 (B, LPB); Prov. Franz Tamayo, Río Bilipisa, $c a 10 \mathrm{~km} \mathrm{NW}$ de Apolo, $1100 \mathrm{~m}, 14^{\circ} 36^{\prime} \mathrm{S}, 6^{\circ} 27^{\prime} \mathrm{W}$, bosque semideciduo disturbado, on bark, 4 July 1997, KB 235 (LPB); DePt. SANTA CruZ. Prov. Florida, Refugio Los Volcanes, $1000 \mathrm{~m}, 18^{\circ} 06^{\prime} \mathrm{S}, 63^{\circ} 36^{\prime} \mathrm{W}$, bosque semi-deciduo, on bark, 3 Oct. 1997, KB et al. 628 (B, LPB).

\section{Teloschistes hypoglaucus (Nyl.) Zahlbr.}

Previously known in Bolivia only from historical data (Nylander 1860, 1861; Malme 1926; Frödén \& Kärnefelt 2007).

Specimens examined: BOLIVIA. Dept. Cochaвамва. Prov. Ayopaya, comunidad de Saila Pata, $2800 \mathrm{~m}, 16^{\circ} 54^{\prime} \mathrm{S}, 66^{\circ} 56^{\prime} \mathrm{W}$, bosque semi-siempreverde, corticolous, 17 Nov. 1997, KB et al. 918 (B, LPB).

\section{Thamnolia papelillo R. Sant.}

Santesson (2004), Flakus (2008) and Flakus et al. (2012a) reported only five localities of this species in Bolivia. The records presented here refer to T. papelillo var. papelillo.

Chemistry. Thamnolic acid.

Specimens examined: BOliVia. Dept. La Paz. Prov. Murillo, below Potosí, $16^{\circ} 17^{\prime} 43^{\prime \prime} \mathrm{S}, 68^{\circ} 07^{\prime} 42^{\prime \prime} \mathrm{W}$, $4720 \mathrm{~m}$, high Andean vegetation, terricolous, 28 May 2011, MK 9503 (LPB, UGDA); Valle del Zongo, Laguna Viscachani, $16^{\circ} 11^{\prime} 54^{\prime \prime} \mathrm{S}, 68^{\circ} 07^{\prime} 33^{\prime \prime} \mathrm{W}, 3860 \mathrm{~m}$, Páramo Yungueño, on soil, 30 May 2011, MK 9660 (LPB, UGDA).

\section{Toninia hosseusiana Gyeln.}

In Bolivia previously reported from only two localities by Flakus and Wilk (2006) and Flakus et al. (2012a).

Specimens examined: BOLIVIA. Dept. La Paz. Prov. Camacho, cerca Ulla Ulla, en el cerro Socondori Grande, $4600 \mathrm{~m}$, vegetación altoandina, terricolous, 4 Dec. 2007, PR 255 (B, LPB).

\section{Trapeliopsis flexuosa (Fr.) Coppins \& P. James}

Only two localities reported from Bolivia (Flakus \& Wilk 2006; Flakus 2008). 
Specimens examined: BOLIVIA. Dept. La PaZ. Prov. Nor Yungas, COTAPATA, by Sillu Tincara preColumbian route, $16^{\circ} 16^{\prime} 33^{\prime \prime} \mathrm{S}, 67^{\circ} 52^{\prime} 60^{\prime \prime} \mathrm{W}, 3429 \mathrm{~m}$, Yungas cloud forest, on twig and bryophytes, 27 June 2010, AF 16987.1 \& PR (LPB).

\section{Umbilicaria cinereorufescens (Schaer.) Frey}

Known in Bolivia from four records (Hestmark 2009; Flakus et al. 2012a).

Specimens examined: BOLIVIA. Dept. Oruro. Prov. Sajama, SAJAMA, Ladera de la Quebrada Kohiri, $4406 \mathrm{~m}, 18^{\circ} 07.132^{\prime} \mathrm{S}, 68^{\circ} 56.088^{\prime} \mathrm{W}$, Polylepis tarapacana forest with large boulders, saxicolous, 21 Nov. 2007, RIM et al. 4397 (B, LPB).

Umbilicaria decussata (Vill.) Zahlbr.

In Bolivia known previously from four localities (Hestmark 2009; Krzewicka 2010; Krzewicka \& Flakus 2010).

Specimens examined: BOliVia. Dept. La Paz. Prov. Camacho, cerca Ulla Ulla, $4770 \mathrm{~m}, 15^{\circ} 01.615^{\prime} \mathrm{S}$, $69^{\circ} 11.882^{\prime} \mathrm{W}$, vegetación altoandina, terricolous, $4 \mathrm{Dec}$. 2007, PR 288 (B, LPB).

\section{Umbilicaria haplocarpa Nyl.}

Reported in several papers (Nylander 1860, 1861; Frey 1936; Almborn 1987; Kärnefelt 1990; Hestmark 2009; Krzewicka \& Flakus 2010) but under-recorded in Bolivia.

Specimens examined: BOLIVIA. Dept. Oruro. Prov. Sajama, SAJAMA, Ladera de la Quebrada Kohiri. 4309 m, $18^{\circ} 07^{\prime} 07^{\prime \prime} \mathrm{S}, 68^{\circ} 56^{\prime} 06^{\prime \prime} \mathrm{W}$, bosque de Polylepis tarapacana, terricolous, 21 Nov. 2007, RIM et al. 4370 (B, LPB).

\section{Umbilicaria leprosa (Zahlbr.) Frey}

In Bolivia known previously from only three localities (Hestmark 2009; Krzewicka \& Flakus 2010).

Specimens examined: Dept. La Paz. Prov. Camacho, near Ulla Ulla, $4739 \mathrm{~m}, 15^{\circ} 02.068^{\prime} \mathrm{S}, 69^{\circ} 11.878^{\prime} \mathrm{W}$, vegetación altoandina, terricolous, 4 Dec. 2007, $P R 267$ (B, LPB); ibid., $4770 \mathrm{~m}, 15^{\circ} 01.615^{\prime} \mathrm{S}, 69^{\circ} 11.882^{\prime} \mathrm{W}$, vegetación altoandina, terricolous, 4 Dec. 2007, $P R$ 286 (B, LPB); Dept. Oruro. Prov. Sajama, SAJAMA, Ladera de la Quebrada Kohiri, 4309 m, $18^{\circ} 07^{\prime} 07^{\prime \prime} \mathrm{S}$, $68^{\circ} 56^{\prime} 06^{\prime \prime} \mathrm{W}$, Polylepis tarapacana forest, saxicolous, 21 Nov. 2007, RIM et al. 4377 (B, LPB).

\section{Umbilicaria polyphylla (L.) Baumg.}

This is the second recent record of the species in Bolivia (Nylander 1859; Frey 1936; Hestmark 2009).

Specimens examined: BOLIVIA. Dept. La Paz. Prov. Camacho, cerca Ulla Ulla, en el cerro Wilamuku, $4739 \mathrm{~m}, 15^{\circ} 02.068^{\prime} \mathrm{S}, 69^{\circ} 11.878^{\prime} \mathrm{W}$, vegetación altoandina, terricolous, 4 Dec. 2007, PR 268 (B, LPB).

Xanthomendoza mendozae (Räsänen) S. Kond. \& Kärnefelt

Previously reported from Bolivia by Kondratyuk and Kärnefelt (1997), Canseco et al. (2006) and Flakus et al. (2012a).

Specimens examined: BOliVia. Dept. La Paz. Prov. Murillo, La Paz, Plaza Abaroa, 3563 m, 16 29.3'S, $68^{\circ} 07.4^{\prime} \mathrm{W}$, on Acacia retinoides, 2 July 2008, G. YapuAlcázar 213 (B, LPB); Plaza Lira, Zona de Sopocachi, $3687 \mathrm{~m}, 16^{\circ} 30.6^{\prime} \mathrm{S}, 68^{\circ} 08.0^{\prime} \mathrm{W}$, on Ulmus pumila, 28 June 2008, G. Yapu-Alcázar 76 (B, LPB); Plaza San Martin, $3560 \mathrm{~m}, 16^{\circ} 30.5^{\prime} \mathrm{S}, 68^{\circ} 07.1^{\prime} \mathrm{W}$, on $U$. pumila. 30 June 2008, G. Yapu-Alcázar 149 (B, LPB).

ACKNOWLEDGEMENTS. We are greatly indebted to Herbario Nacional de Bolivia, Universidad Mayor de San Andrés and Museo Nacional de Historia Natural La Paz for their generous cooperation, the Curators of herbaria for the loan of specimens, and our colleagues and the staff of all the protected areas for their kind help during fieldwork. Also, we are grateful to Professor Mark R. D. Seaward (Bradford, UK) and the anonymous reviewer for helpful suggestions on the manuscript. This research received financial support from the Polish National Centre for Research and Development (NCBiR) under the LIDER Programme for 2010-2013 (No. 92/L-1/09), and from the W. Szafer Institute of Botany of the Polish Academy of Sciences, through its statutory fund.

\section{REFERENCES}

Almborn O. 1987. Lichens at high altitudes in Southern Africa. Biblioth. Lichenol. 25: 401-417.

Aptroot A. \& Bungartz F. 2007. The lichen genus Ramalina on the Galapagos. Lichenologist 36: 519-542. 
Aptroot A. \& Sparrius L. B. 2003. New microlichens from Taiwan. Fungal Div. 14: 1-50.

Awasthi D. D. 1975. A monograph of the lichen genus Dirinaria. Biblioth. Lichenol. 2: 1-108.

Boom VAN DEN P., Elix J. A. \& Sipman H. J. M. 2007. New or interesting lichen records from Guatemala I. Willdenowia 37: 363-375.

Breuss O. \& Neuwirth G. 2007. Flechtenfunde im Bosque Esquinas, Costa Rica. Linzer Biol. Beitr. 39(1): 557-569.

Bungartz F., Ziemmeck F., Yánez Ayabaca A., Nugra F. \& Aptroot A. 2013. CDF Checklist of Galapagos Lichenized Fungi. FCD Lista de especies de Hongos liquenizados Galápagos. (Last updated: 6 Jun 2013). http:// checklists.datazone.darwinfoundation.org/true-fungi/ lichens.

Cáceres M. E. D. S., Dos Santos V. M., De Góes D. T., Mota D. A. \& Aptroot A. 2013. Two new species of Malmidea from north-eastern Brazil. Lichenologist 45: 619-622.

Calvelo S. \& Liberatore S. 2002. Catálogo de los líquenes de la Argentina. Kurtziana 29(2): 7-170.

Canseco A., Anze R. \& Franken M. 2006. Comunidades de liquenes: indicadores de la calidad del aire en la ciudad de La Paz, Bolivia. Acta Nova 3(2): 286-307.

Culberson C. F. \& Kristinsson H. 1970. A standardized method for the identification of lichen products. J. Chromatogr. 46: 85-93.

Elix J. A. \& Nash T. H. 1992: A synopsis of the lichen genus Psiloparmelia (Ascomycotina, Parmeliaceae). Bryologist 95: 377-391.

EsSLINGER T. L. 2014. A cumulative checklist for the lichenforming, lichenicolous and allied fungi of the continental United States and Canada. North Dakota State University, Fargo, North Dakota. [Version (\#19) 23 March 2014]. http://www.ndsu.edu/pubweb/ esslinge/chcklst/chcklst7. htm.

Feuerer T. \& Sipman H. J. M. 2005. Additions to the lichenized and lichenicolous fungi of Bolivia. Herzogia 18: 139-144.

Feuerer T., Ahti T. \& Vitikainen O. 1998. Lichenological investigations in Bolivia. In: M. P. MARCelli \& M. R. D. SEAWARD (eds), Lichenology in Latin America: history, current knowledge and applications, pp. 71-86. CETESB, Săo Paulo.

Flakus A. 2008. Contributions to the knowledge of the lichen biota of Bolivia. 2. Polish Bot. J. 53: 145-153.

Flakus A. \& KuKwa M. 2007. New species and records of Lepraria (Stereocaulaceae, lichenized Ascomycota) from South America. Lichenologist 39: 463-474.

Flakus A. \& Wilk K. 2006. Contribution to the knowledge of the lichen biota of Bolivia. J. Hattori Bot. Lab. 99: 307-318.

Flakus A., Elix J. A., Rodriguez P. \& Kukwa M. 2011 a. New species and records of Lepraria (Stereocaulaceae, lichenized Ascomycota) from South America. Lichenologist 43: 57-66.

Flakus A., Oset M., Jablońska A., Rodriguez SaAvedra P. \& KuKwa M. 2011b. Contribution to the knowledge of the lichen biota of Bolivia. 3. Polish Bot. J. 56: 159-183.

Flakus A., Etayo J., Schiefelbein U., Ahti T., JablońsKa A., Oset M., Bach K., Rodriguez Flakus P. \& Kukwa M. 2012a. Contribution to the knowledge of the lichen biota of Bolivia. 4. Polish Bot. J. 57: 427-461.

Flakus A., Rodriguez SaAvedra P. \& Kukwa M. 2012 b. A new species and new combinations and records of $\mathrm{Hy}$ potrachyna and Remototrachyna from Bolivia. Mycotaxon 119: $157-166$.

Flakus A., Sipman H. J. M., Bach K., Rodriguez Flakus P., Knudsen K., Ahti T., Schiefelbein U., Palice Z., Jablońska A., Oset M., Meneses Q. R. I. \& KuKwa M. 2013. Contribution to the knowledge of the lichen biota of Bolivia. 5. Polish Bot. J. 58: 697-733.

Flakus A., Sipman H. J. M., Rodriguez Flakus P., SchieFelbein U., JablońsKa A., Oset M. \& Kukwa M. 2014. Contribution to the knowledge of the lichen biota of Bolivia. 6. Polish Bot. J. 59: 63-83.

FreY E. 1936. Die geographische Verbreitung der Umbilicariaceen und einiger alpiner Flechten. Ber. Schweiz. Bot. Ges. 46: 412-444.

FrödÉn P. \& KÄRnefelt I. 2007. Two new species of Teloschistes J. M. Norman: T. arabicus and T. inflatus and notes on the Teloschistes flora of Africa. Biblioth. Lichenol. 95: 183-223.

FrödÉN P. \& LindBlom L. 2003. Josefpoeltia parva, a new combination in Josefpoeltia (Teloschistaceae). Bryologist 106: $447-450$.

Galloway D. J. \& Quilhot W. 1998. Checklist of Chilean lichen-forming and lichenicolous fungi. Gayana, Bot. 55: 111-185.

Hale M. E. 1989. A new lichen genus, Psiloparmelia Hale (Ascomycotina: Parmeliaceae). Mycotaxon 35: 41-44.

Hale M. E. \& Fletcher A. 1990. Rimelia Hale \& Fletcher, a new lichen genus (Ascomycotina: Parmeliaceae). Bryologist 93: 23-29.

Herzog T. 1922. Beitrag zur Flechtenflora von Bolivia. Hedwigia 63: 263-268.

Hestmark G. 2009. New observations and records for Umbilicaria (Umbilicariaceae) in Bolivia. Bryologist 112: 833-838.

Josse C., Navarro G., Comer P., Evans R., Faber-Langendoen D., Fellows M., Kittel G., Menard S., Pyne M., Reid M., Schulz K., Snow K. \& Teague J. 2003. Ecological Systems of Latin America and the Caribbean: A Working Classification of Terrestrial Systems. NatureServe, Arlington. http://www.natureserve.org/library/ LACEcologicalSystems.pdf 
KALB K. 1996. Additional lichen records from Australia. 29. Australas. Lichenol. Newslett. 39: 28-34.

KALB K. \& V̌̌ZdA A. 1988. Neue oder bemerkenswerte Arten der Flechtenfamilie Gomphillaceae in der Neotropis. Biblioth. Lichenol. 29: 1-80.

KantviLas G. 1998. Studies on the lichen genus Siphula in Tasmania II. The $S$. decumbens group. Herzogia 13: 119-138.

Kantvilas G. \& Elix J. E. 2002. The taxonomy, chemistry and morphology of some South American species of Siphula. Herzogia 15: 1-12.

KÄRNEFELT I. 1990. Evidence of a slow evolutionary change in the speciation of lichens. Biblioth. Lichenol. 38: 291-306.

Kashiwadani H. 1987. Peruvian species of Ramalina (lichens). In: H. Inoue (ed.), Studies on Cryptogams in Southern Peru, pp. 129-144. Tokai University Press, Tokyo.

Kashiwadani H. \& Kalb K. 1993. The genus Ramalina in Brazil. Lichenologist 25: 1-31.

KondratyuK S. \& Kärnefelt I. 1997. Josefpoeltia and Xanthomendoza, two new genera in the Teloschistaceae (lichenized Ascomycotina). Biblioth. Lichenol. 68: 19-44.

Krog H. \& Swinscow T. D. V. 1976. The genus Ramalina in East Africa. Norweg. J. Bot. 23: 153-175.

KrZEWICKA B. 2010. Umbilicaria rhizinata comb. nov. (1ichenized Ascomycota). Lichenologist 42: 491-493.

Krzewicka B. \& Flakus A. 2010. New records of the genus Umbilicaria (Umbilicariaceae, lichenized Ascomycota) from Bolivia. Cryptog. Mycol. 31: 441-451.

Kukwa M. \& Flakus A. 2009. Lepraria glaucosorediata sp. nov. (Stereocaulacae, lichenized Ascomycota) and other interesting records of Lepraria. Mycotaxon 108: 353-364.

Kukwa M., Bach K., Sipman H. J. M. \& Flakus A. 2012. Thirty-six species of the lichen genus Parmotrema (Lecanorales, Ascomycota) new to Bolivia. Polish Bot. J. 57: 243-257.

LAMB I. M. 1977. A conspectus of the lichen genus Stereocaulon (Schreb.) Hoffm. J. Hattori Bot. Lab. 43: 191-355.

Lendemer J. C. 2013. Molecular phylogenetic evidence corroborates morphology but not chemistry in the Lepraria neglecta group (Stereocaulaceae). Mem. New York Bot. Gard. 108: 127-153.

LóPEz-FigueIras M. 1986. Censo de macrolíquenes venezolanos de los estados Falcon, Lara, Merida, Tachira y Trujillo. Facultad de Farmacia, Universidad de Los Andes, Merida.

LÜCKIng R., Buck W. R. \& Riva Plata E. 2007. The lichen family Gomphillaceae (Ostropales) in eastern North America, with notes on hyphophore development in Gomphillus and Gyalideopsis. Bryologist 110: 622-672

LyNGE B. 1925. On Some South American Lichens of the Genera Parmelia, Candelaria, Telochistes and Pyxine. Nyt Mag. Naturvidensk. 62: 83-87.
Malme G. O. A. 1926. Lichenes blasteniospori Herbarii Regnelliani. Ark. Bot. 20A(9): 1-51.

Marcano V. \& Morales A. 1994. A new species of Ramalina from the Venezuelan Andes. Ernstia 3: 101-104.

Marcelli M. P., Canęz L. S., Benatti M. N., Spielmann A. A., Jungbluth P. \& Elix J. A. 2011. Taxonomical novelties in Parmeliaceae. Biblioth. Lichenol. 106: 211-224.

McCarthy P. M. 2014. Checklist of the Lichens of Australia and its Island Territories. Version 27 June 2014. Canberra: Australian Biological Resources Study. http://www.anbg. gov.au/abrs/lichenlist/introduction.html.

Moberg R. 1990. The lichen genus Physcia in Central and South America. Nord. J. Bot. 10: 319-342.

Moberg R. 2011. The lichen genus Heterodermia (Physciaceae) in South America - a contribution including five new species. Nord. J. Bot. 29: 129-147.

Myers N., Mittermeier R. A., Mittermeier C. G., Da FonSECA G. A. B. \& Kent J. 2000. Biodiversity hotspots for conservation priorities. Nature 403: 853-858.

NYLANDER W. 1859. Lichenes in regionibus exoticis quibusdam vigentes exponit synopticis enumerationibus. Ann. Sci. Nat. Bot. 11: 205-264.

NyLANDER W. 1860. Synopsis methodica lichenum. Fasciculus I. Tomus primus. L. Martinet, Paris.

NYLANDER W. 1861. Additamentum ad lichenographiam Andium Boliviensium. Ann. Sci. Nat. Bot. 15: 365-382.

Orange A., James P. W. \& White F. J. 2001. Microchemical methods for the identification of lichens. British Lichen Society, London.

Oset M. \& Kukwa M. 2012. Notes on Stereocaulon species from Bolivia. Mycotaxon 121: 447-453.

Poelt J. 1974. Zur Kenntnis der Flechtenfamilie Candelariaceae. Ein Beitrag mit besonderer Berücksichtigung einiger südamerikanischer Arten. Phyton 16: 189-210.

Quilhot W., Rubio C. \& Cuellar M. 2007. Comparative studies between the lichen flora from Chile and Antarctica. Biblioth.Lichenol. 95: 479-488.

Rodriguez Flakus P., Flakus A., Kukwa M., Etayo J., LÜCKing R., Isela Meneses R., Rivas Plata E., Stanton D., TruOng C. \& VARgas R. 2014. Preliminary catalogue of lichens and lichenicolous fungi from Bolivia. W. Szafer Institute of Botany PAS, Krakow. [Version 1.4 (1 February 2014)]. http://botany.pl/lichens-bolivia.

RusBy H. H. 1896. An enumeration of the plants collected in Bolivia by Miguel Bang, with description of new genera and species. Part III. Mem. Torrey Bot. Club 6: 1-130.

Santesson R. 2004. Two new species of Thamnolia. Symb. Bot. Upsal. 34(1): 393-397.

Sipman H. J. M. 1992. The origin of the lichen flora of the Colombian páramos. In: H. BALSLEv \& J. L. LuteYN (eds), 
Páramo, Andean ecosystem under human influence, pp. 95-109. Academic Press, London.

Sipman H. J. M. 2002. Key to the Stereocaulon species in the Neotropics. (Version 27 Aug. 2002). http://www.bgbm. org/sipman/keys/Neostereo.htm.

SiPMAN H. J. M. 2006. Diversity and biogeography of lichens in Neotropical Montane Oak Forests. In: M. KAPPELLE (ed.), Ecology and conservation of Neotropical Montane Oak Forests, pp. 69-81. Springer-Verlag, Berlin, Heidelber.

Sipman H. J. M. \& Wolf J. H. D. 1998. Provisional checklist for the lichens of Chiapas. Acta Bot. Mexicana 45: 1-29.

Sipman H. J. M., Hekking W. \& Aguirre-C. J. 2008. Checklist of lichenized and lichenicolous fungi from Colombia. Biblioteca José Jerónimo Triana 20. Instituto de Ciencias Naturales, Facultad de Ciencias, Universidad Nacional de Colombia, Bogotá.

Søchting U. \& FröDÉn P. 2002. Chemosyndromes in the lichen genus Teloschistes (Teloschistaceae, Lecanorales). Mycol. Progr. 1: 257-266.

Spielmann A. A. 2009. Estudos taxonômicos em Parmotrema s.1. (Parmeliaceae, Ascomycota liquenizados) com ácido salazínico. PhD Thesis, Instituto de Botânica da Secretaria de Estado do Meio Ambiente, Săo Paulo.

Thell A., Feuerer T., Elix J. A. \& Kärnefelt I. 2006. A contribution to the phylogeny and taxonomy of Xanthoparmelia (Ascomycota, Parmeliaceae). J. Hattori Bot. Lab. 100: $797-807$.
Thell A., Feuerer T., Kärnefelt I., Myllys L. \& Stenroos S. 2004. Monophyletic groups within the Parmeliaceae identified by ITS rDNA, $\beta$-tubulin and GAPDH sequences. Mycol. Progr. 3: 297-314.

ThIERs B. 2012. Index Herbariorum: A global directory of public herbaria and associated staff. New York Botanical Garden's Virtual Herbarium. http://sweetgum.nybg.org/ih.

Tucker S. C. 1981. Checklist of Louisiana lichens. Proc. Louisiana Acad. Sci. 44: 58-70.

VAINIO E. A. 1890. Étude sur la classification naturelle et la morphologie des Lichens du Brésil. Pars prima. Acta Soc. Fauna Fl. Fenn. 7(1): i-xxix + 1-247.

VĚZDA A. 1966. Flechtensystematische Studien III. Die Gattungen Ramonia Stiz. und Gloeolecta Lett. Folia Geobot. Phytotax. 1: 154-175.

VĚZDA A. 1979. Flechten systematische Studien XI. Beitrage zur Kenntnis der Familie Asterothyriaceae (Discolichens). Folia Geobot. Phytotax. 14: 43-94.

Westberg M., Fröden P. \& Wedin M. 2009. A monograph of the genus Placomaronea (Ascomycota, Candelariales). Lichenologist 41: 513-527.

WILK K. 2010. Icmadophila aversa and Piccolia conspersa, two lichen species new to Bolivia. Polish Bot. J. 55: 217-221.

ZAHLBRUCKNER A. 1902. Studien über brasilianische Flechten. Sitzungsber. Kaiserl. Akad. Wiss., Wien, Math.-Naturwiss. Cl., Abt. 1 111(1): 357-432 + Tafel I-II. 